Research paper

\title{
Nonlocal modified KdV equations and their soliton solutions by Hirota Method
}

\author{
Metin Gürses ${ }^{\mathrm{a}}$, Aslı Pekcan ${ }^{\mathrm{b}, *}$ \\ a Department of Mathematics, Faculty of Science Bilkent University, Ankara 06800, Turkey \\ ${ }^{\mathrm{b}}$ Department of Mathematics, Faculty of Science Hacettepe University, Ankara 06800, Turkey
}

\section{A R T I C L E I N F O}

\section{Article history:}

Received 24 March 2018

Accepted 9 July 2018

Available online 20 July 2018

\section{Keywords:}

Ablowitz-Musslimani reduction

Nonlocal mKdV equations

Hirota bilinear form

Soliton solutions

\begin{abstract}
A B S T R A C T
We study the nonlocal modified Korteweg-de Vries (mKdV) equations obtained from AKNS scheme by Ablowitz-Musslimani type nonlocal reductions. We first find soliton solutions of the coupled mKdV system by using the Hirota direct method. Then by using the Ablowitz-Musslimani reduction formulas, we find one-, two-, and three-soliton solutions of nonlocal $\mathrm{mKdV}$ and nonlocal complex mKdV equations. The soliton solutions of these equations are of two types. We give one-soliton solutions of both types and present only first type of two- and three-soliton solutions. We illustrate our solutions by plotting their graphs for particular values of the parameters.
\end{abstract}

(c) 2018 Elsevier B.V. All rights reserved.

\section{Introduction}

Nonlocal integrable equations studied so far are of integro-differential equation type, such as Benjamin-Ono equation. Recently [1] Ablowitz and Musslimani have introduced a new type of nonlocal integrable equations. In these new types of nonlocal equations, in addition to the terms at the space-time point $(t, x)$, there are terms at the mirror image point $(t,-x)$. All such new integrable equations seem to be obtained by a reduction from an integrable system of coupled integrable equations. For instance when the Lax pair is a cubic polynomial of the spectral parameter we obtain coupled modified Korteweg-de Vries system of equations from the AKNS formalism [6]. These equations are given by

$$
\begin{aligned}
& a q_{t}=-\frac{1}{4} q_{x x x}+\frac{3}{2} r q q_{x}, \\
& a r_{t}=-\frac{1}{4} r_{x x x}+\frac{3}{2} r q r_{x},
\end{aligned}
$$

where $q(t, x)$ and $r(t, x)$ are in general complex dynamical variables, $a$ is a constant. We call the above system of coupled equations as nonlinear modified Korteweg-de Vries system (mKdV system). We have two different local (standard) reductions of this system:

$$
\text { a) } r(t, x)=k \bar{q}(t, x)
$$

\footnotetext{
* Corresponding author.

E-mail addresses: gurses@fen.bilkent.edu.tr (M. Gürses), aslipekcan@hacettepe.edu.tr (A. Pekcan).
} 


$$
\text { b) } r(t, x)=k q(t, x) \text {, }
$$

where $k$ is a real constant and $\bar{q}$ is the complex conjugate of the function $q$. When we apply the reduction (3) to the Eqs. (1) and (2) we obtain the complex modified Korteweg-de Vries (cmKdV) equation [2-4]

$$
a q_{t}=-\frac{1}{4} q_{x x x}+\frac{3}{2} k \bar{q} q q_{x}
$$

provided that $\bar{a}=a$. The second reduction (4) gives the usual mKdV equation [5]

$$
a q_{t}=-\frac{1}{4} q_{x x x}+\frac{3}{2} k q^{2} q_{x}
$$

with no condition on $a$.

Most of the integrable nonlinear equations are local that is the solution's behavior depends only on its local space and time parameters. In $[1,7,8]$ Ablowitz and Musslimani introduced integrable nonlocal reductions which yield the spacetime reflection symmetric (ST-symmetric), the space reflection symmetric (S-symmetric), and time reflection symmetric (Tsymmetric) equations. For instance in the S-symmetric case, the solution's behavior at location $(t, x)$ depends on the information not only at the point $(t, x)$ but also at the point $(t,-x)$. Ablowitz and Musslimani introduced the ST-symmetric and $\mathrm{T}$-symmetric nonlocal $\mathrm{mKdV}$ and $\mathrm{cmKdV}$ equations and they only obtained one-soliton solutions of ST-symmetric ones by using inverse scattering transform in [8]. A nonlocal reduction is given by

$$
r(t, x)=k \bar{q}\left(\varepsilon_{1} t, \varepsilon_{2} x\right)
$$

where $\varepsilon_{1}^{2}=\varepsilon_{2}^{2}=1$. Under this condition the mKdV system (1) and (2) reduce to

$$
a q_{t}(t, x)=-\frac{1}{4} q_{x x x}(t, x)+\frac{3}{2} k \bar{q}\left(\varepsilon_{1} t, \varepsilon_{2} x\right) q(t, x) q_{x}(t, x),
$$

provided that $\bar{a}=\varepsilon_{1} \varepsilon_{2} a$. The case for $\left(\varepsilon_{1}, \varepsilon_{2}\right)=(1,1)$ yields the local equation (5). There are three different nonlocal reductions where $\left(\varepsilon_{1}, \varepsilon_{2}\right)=\{(-1,1),(1,-1),(-1,-1)\}$. Hence for these values of $\varepsilon_{1}$ and $\varepsilon_{2}$ and for different signs of $k$ $(\operatorname{sign}(k)= \pm 1)$, we have six different nonlocal integrable cmKdV equations obtained by Ablowitz-Musslimani type reduction (7) which are respectively T-symmetric, S-symmetric, and ST-symmetric nonlocal cmKdV equations given below in part A.

A. $r(t, x)=k \bar{q}\left(\varepsilon_{1} t, \varepsilon_{2} x\right)$ (Nonlocal cmKdV equations)

1. T-symmetric cmKdV equation:

$$
a q_{t}(t, x)=-\frac{1}{4} q_{x x x}(t, x)+\frac{3}{2} k \bar{q}(-t, x) q(t, x) q_{x}(t, x), \quad \bar{a}=-a .
$$

2. S-symmetric cmKdV equation:

$$
a q_{t}(t, x)=-\frac{1}{4} q_{x x x}(t, x)+\frac{3}{2} k \bar{q}(t,-x) q(t, x) q_{x}(t, x), \quad \bar{a}=-a .
$$

3. ST-symmetric cmKdV equation:

$$
a q_{t}(t, x)=-\frac{1}{4} q_{x x x}(t, x)+\frac{3}{2} k \bar{q}(-t,-x) q(t, x) q_{x}(t, x), \quad \bar{a}=a .
$$

The second nonlocal reduction of the mKdV system is given by

$$
r(t, x)=k q\left(\varepsilon_{1} t, \varepsilon_{2} x\right),
$$

yielding the equation

$$
a q_{t}(t, x)=-\frac{1}{4} q_{x x x}(t, x)+\frac{3}{2} k q(t, x) q\left(\varepsilon_{1} t, \varepsilon_{2} x\right) q_{x}(t, x),
$$

provided that $\varepsilon_{1} \varepsilon_{2}=1$. Therefore we have only one possibility $\left(\varepsilon_{1}, \varepsilon_{2}\right)=(-1,-1)$ to have a nonlocal equation, without any additional condition on the parameter $a$. The ST-symmetric nonlocal mKdV equation obtained here is given below in part $B$.

B. $r(t, x)=k q\left(\varepsilon_{1} t, \varepsilon_{2} x\right)$ (Nonlocal mKdV equation)

1. ST-symmetric mKdV equation:

$$
a q_{t}(t, x)=-\frac{1}{4} q_{x x x}(t, x)+\frac{3}{2} k q(-t,-x) q(t, x) q_{x}(t, x),
$$

with no condition on $a$. 
The nonlocal cmKdV and nonlocal mKdV equations have the focusing and defocusing cases when $k<0$ and $k>0$, respectively. All the above equations are integrable.

There is an increasing interest in obtaining the nonlocal reductions of systems of integrable equations and analyzing their solutions and properties [9-24] after Ablowitz and Musslimani's works [1] and [7,8] in which they proposed many nonlocal nonlinear integrable equations such as nonlocal nonlinear Schrödinger (NLS) equation, cmKdV and mKdV equations, sineGordon equation, $(1+1)$ and $(2+1)$ dimensional three-wave interaction, Davey-Stewartson equation, and derivative NLS equation. They discussed Lax pairs, conservation laws, inverse scattering transforms, and obtained one-soliton solutions of some of these equations. In Ref. [25], Ma et al. showed that ST-symmetric nonlocal cmKdV equation is gauge equivalent to a spin-like model which shows that there exists significant difference between the nonlocal cmKdV and the local cmKdV equation. They constructed Darboux transformations for nonlocal cmKdV and obtained different type of exact solutions including dark-soliton, W-type soliton, M-type soliton, and periodic solutions. Ji and Zhu obtained soliton, kink, anti-kink, complexiton, breather, rogue-wave solutions, and nonlocalized solutions with singularities of ST-symmetric nonlocal mKdV equation through Darboux transformation and inverse scattering transform [26,27]. In [28], the authors showed that many nonlocal integrable equations, such as Davey-Stewartson equation, T-symmetric NLS equation, nonlocal derivative NLS equation, and ST-symmetric cmKdV equation can be converted to local integrable equations by simple variable transformations. They used these transformations to obtain solutions of the nonlocal equations from the solutions of the local equations and to derive new nonlocal integrable equations, such as complex and real ST- and T-symmetric NLS equations and nonlocal complex short pulse equations. For some possible application of nonlocal NLS and nonlocal mKdV equations one can check [29-31]. Some of our solutions coincide with the solutions given in $[8,25,27,28]$.

The main purpose of this work is to search for possible integrable nonlocal reductions of the mKdV system (1) and (2) and find their soliton solutions by the application of the Hirota direct method. To find the soliton solutions of the nonlocal mKdV and nonlocal cmKdV equations we first find soliton solutions of the mKdV system (1) and (2). By using the reduction formulas (7) and (12) we find the soliton solutions of the nonlocal mKdV and nonlocal cmKdV equations. Actually, here we introduce a general method for finding soliton solutions of nonlocal integrable equations. If a nonlocal equation is consistently obtained by a nonlocal reduction of a system of equations having soliton solutions either by Hirota direct method or by other techniques, such as the inverse scattering transform technique, one can automatically use the reduction formulas (constraint equations) to find the soliton solutions of the reduced nonlocal equations.

In a previous paper [32], we have studied the soliton solutions of the NLS system and nonlocal NLS equations. In this work, we study soliton solutions of the nonlocal mKdV equations of all types. Following the work of Iwao and Hirota [2] we first find one-, two-, and three-soliton solutions of the coupled mKdV system (1) and (2) by using the Hirota direct method. Then by using the Ablowitz-Musslimani type reductions (7) and (12), we obtain soliton solutions of the nonlocal cmKdV (including T-, S-, and ST-symmetric equations) and nonlocal ST-symmetric mKdV equations. We show that there are two different types of one-soliton solution of the reduced $\mathrm{mKdV}$ system. We give the corresponding two- and three-soliton solutions of the first type. We also present the graphs of some solutions for certain values of the parameters. They include one-, two-, and three-soliton waves, complexitons, breather-type, and kink-type waves.

The lay out of the paper is as follows. In Section 2 we apply Hirota method to the coupled mKdV system (1) and (2) and find soliton solutions. In Section 3 we find soliton solutions of T-symmetric, S-symmetric, and two different ST-symmetric mKdV equations and we give some examples for one-, two-, and three-soliton solutions together with their graphs.

\section{Hirota method for the coupled MKdV system}

Following the work of Iwao and Hirota [2] let $q=\frac{F}{f}$ and $r=\frac{G}{f}$. Eq. (1) becomes

$$
\begin{gathered}
4 a F_{t} f^{3}-4 a F f_{t} f^{2}+F_{x x x} f^{3}-3 F_{x x} f_{x} f^{2}+6 F_{x} f_{x}^{2} f-3 F_{x} f_{x x} f^{2} \\
-6 F f_{x}^{3}+6 F f_{x} f_{x x} f-F f_{x x x} f^{2}-6 G F F_{x} f+6 G F^{2} f_{x}=0,
\end{gathered}
$$

which is equivalent to

$$
f^{2}\left(4 a D_{t}+D_{x}^{3}\right) F \cdot f-3\left(D_{x}^{2} f \cdot f+2 G F\right)\left(D_{x} F \cdot f\right)=0 .
$$

Similarly, the Eq. (2) can be written as

$$
f^{2}\left(4 a D_{t}+D_{x}^{3}\right) G \cdot f-3\left(D_{x}^{2} f \cdot f+2 G F\right)\left(D_{x} G \cdot f\right)=0 .
$$

Hence the Hirota bilinear form of the mKdV system is

$$
\begin{aligned}
& P_{1}(D)\{F \cdot f\} \equiv\left(4 a D_{t}+D_{x}^{3}-3 \alpha D_{x}\right)\{F \cdot f\}=0 \\
& P_{2}(D)\{G \cdot f\} \equiv\left(4 a D_{t}+D_{x}^{3}-3 \alpha D_{x}\right)\{G \cdot f\}=0 \\
& P_{3}(D)\{f \cdot f\} \equiv\left(D_{x}^{2}-\alpha\right)\{f \cdot f\}=-2 G F,
\end{aligned}
$$

where $\alpha$ is an arbitrary constant. Note that for mKdV system we obtain similar solutions as in the NLS system case [32]. For a detailed work of the application of the Hirota method to the mKdV system one can check [33]. 
2.1. One-soliton solution of the MKdV system

To find one-soliton solution we use the following expansions for the functions $F$, $G$, and $f$,

$$
F=\varepsilon F_{1}, \quad G=\varepsilon G_{1}, \quad f=1+\varepsilon^{2} f_{2},
$$

where

$$
F_{1}=e^{\theta_{1}}, \quad G_{1}=e^{\theta_{2}}, \quad \theta_{i}=k_{i} x+\omega_{i} t+\delta_{i}, i=1,2 .
$$

We insert these expansions into (16)-(18). The coefficient of $\varepsilon^{0}$ gives

$$
\left(D_{x}^{2}-\alpha\right)\{1 \cdot 1\}=0
$$

yielding that $\alpha=0$. Analyzing the coefficients of $\varepsilon^{n}, 1 \leq n \leq 4$ give the dispersion relations

$$
\omega_{i}=-\frac{k_{i}^{3}}{4 a}, \quad i=1,2,
$$

and the function $f_{2}$

$$
f_{2}=-\frac{e^{\left(k_{1}+k_{2}\right) x+\left(\omega_{1}+\omega_{2}\right) t+\delta_{1}+\delta_{2}}}{\left(k_{1}+k_{2}\right)^{2}} .
$$

Take $\varepsilon=1$. Hence a pair of solutions of the mKdV system (1) and (2) is given by $(q(t, x), r(t, x))$ where

$$
q(t, x)=\frac{e^{\theta_{1}}}{1+A e^{\theta_{1}+\theta_{2}}}, \quad r(t, x)=\frac{e^{\theta_{2}}}{1+A e^{\theta_{1}+\theta_{2}}},
$$

with $\theta_{i}=k_{i} x-\frac{k_{i}^{3}}{4 a} t+\delta_{i}, i=1,2$, and $A=-\frac{1}{\left(k_{1}+k_{2}\right)^{2}}$. Here $k_{1}, k_{2}, \delta_{1}$, and $\delta_{2}$ are arbitrary complex numbers.

\subsection{Two-soliton solution of the MKdV system}

For two-soliton solution, we take

$$
F=\varepsilon F_{1}+\varepsilon^{3} F_{3}, \quad G=\varepsilon G_{1}+\varepsilon^{3} G_{3}, \quad f=1+\varepsilon^{2} f_{2}+\varepsilon^{4} f_{4},
$$

where

$$
F_{1}=e^{\theta_{1}}+e^{\theta_{2}}, \quad G_{1}=e^{\eta_{1}}+e^{\eta_{2}},
$$

with $\theta_{i}=k_{i} x+\omega_{i} t+\delta_{i}, \eta_{i}=\ell_{i} x+m_{i} t+\alpha_{i}$ for $i=1$, 2. When we insert above expansions into (16)-(18) and consider the coefficients of $\varepsilon^{n}, 1 \leq n \leq 8$ we obtain the dispersion relations

$$
\omega_{i}=-\frac{k_{i}^{3}}{4 a}, \quad m_{i}=-\frac{\ell_{i}^{3}}{4 a}, \quad i=1,2,
$$

the function $f_{2}$,

$$
f_{2}=e^{\theta_{1}+\eta_{1}+\alpha_{11}}+e^{\theta_{1}+\eta_{2}+\alpha_{12}}+e^{\theta_{2}+\eta_{1}+\alpha_{21}}+e^{\theta_{2}+\eta_{2}+\alpha_{22}}=\sum_{1 \leq i, j \leq 2} e^{\theta_{i}+\eta_{j}+\alpha_{i j}},
$$

where

$$
e^{\alpha_{i j}}=-\frac{1}{\left(k_{i}+\ell_{j}\right)^{2}}, 1 \leq i, j \leq 2,
$$

the functions $F_{3}$ and $G_{3}$,

$$
F_{3}=\gamma_{1} e^{\theta_{1}+\theta_{2}+\eta_{1}}+\gamma_{2} e^{\theta_{1}+\theta_{2}+\eta_{2}}, \quad G_{3}=\beta_{1} e^{\theta_{1}+\eta_{1}+\eta_{2}}+\beta_{2} e^{\theta_{2}+\eta_{1}+\eta_{2}},
$$

where

$$
\gamma_{i}=-\frac{\left(k_{1}-k_{2}\right)^{2}}{\left(k_{1}+\ell_{i}\right)^{2}\left(k_{2}+\ell_{i}\right)^{2}}, \quad \beta_{i}=-\frac{\left(\ell_{1}-\ell_{2}\right)^{2}}{\left(\ell_{1}+k_{i}\right)^{2}\left(\ell_{2}+k_{i}\right)^{2}}, \quad i=1,2,
$$

and the function $f_{4}$

$$
f_{4}=M e^{\theta_{1}+\theta_{2}+\eta_{1}+\eta_{2}}
$$

where

$$
M=\frac{\left(k_{1}-k_{2}\right)^{2}\left(l_{1}-l_{2}\right)^{2}}{\left(k_{1}+l_{1}\right)^{2}\left(k_{1}+l_{2}\right)^{2}\left(k_{2}+l_{1}\right)^{2}\left(k_{2}+l_{2}\right)^{2}} .
$$


Let us also take $\varepsilon=1$. Then two-soliton solution of the mKdV system (1) and (2) is given with the pair $(q(t, x), r(t, x))$,

$$
\begin{aligned}
& q(t, x)=\frac{e^{\theta_{1}}+e^{\theta_{2}}+\gamma_{1} e^{\theta_{1}+\theta_{2}+\eta_{1}}+\gamma_{2} e^{\theta_{1}+\theta_{2}+\eta_{2}}}{1+e^{\theta_{1}+\eta_{1}+\alpha_{11}}+e^{\theta_{1}+\eta_{2}+\alpha_{12}}+e^{\theta_{2}+\eta_{1}+\alpha_{21}}+e^{\theta_{2}+\eta_{2}+\alpha_{22}}+M e^{\theta_{1}+\theta_{2}+\eta_{1}+\eta_{2}}}, \\
& r(t, x)=\frac{e^{\eta_{1}}+e^{\eta_{2}}+\beta_{1} e^{\theta_{1}+\eta_{1}+\eta_{2}}+\beta_{2} e^{\theta_{2}+\eta_{1}+\eta_{2}}}{1+e^{\theta_{1}+\eta_{1}+\alpha_{11}}+e^{\theta_{1}+\eta_{2}+\alpha_{12}}+e^{\theta_{2}+\eta_{1}+\alpha_{21}}+e^{\theta_{2}+\eta_{2}+\alpha_{22}}+M e^{\theta_{1}+\theta_{2}+\eta_{1}+\eta_{2}}},
\end{aligned}
$$

with $\theta_{i}=k_{i} x-\frac{k_{i}^{3}}{4 a} t+\delta_{i}, \eta_{i}=\ell_{i} x-\frac{\ell_{i}^{3}}{4 a} t+\alpha_{i}$ for $i=1,2$. Here $k_{i}, \ell_{i}, \delta_{i}$, and $\alpha_{i}, i=1,2$ are arbitrary complex numbers.

\subsection{Three-soliton solution of the MKdV system}

To find three-soliton solution, we take

$$
f=1+\varepsilon^{2} f_{2}+\varepsilon^{4} f_{4}+\varepsilon^{6} f_{6}, \quad G=\varepsilon G_{1}+\varepsilon^{3} G_{3}+\varepsilon^{5} G_{5}, \quad F=\varepsilon F_{1}+\varepsilon^{3} F_{3}+\varepsilon^{5} F_{5},
$$

and

$$
F_{1}=e^{\theta_{1}}+e^{\theta_{2}}+e^{\theta_{3}}, \quad G_{1}=e^{\eta_{1}}+e^{\eta_{2}}+e^{\eta_{3}},
$$

where $\theta_{i}=k_{i} x+\omega_{i} t+\delta_{i}, \eta_{i}=\ell_{i} x+m_{i} t+\alpha_{i}$ for $i=1,2,3$. Inserting (36) into (16)-(18) and analyzing the coefficients of $\varepsilon^{n}$, $1 \leq n \leq 12$ give the dispersion relations

$$
\omega_{i}=-\frac{k_{i}^{3}}{4 a}, \quad m_{i}=-\frac{\ell_{i}^{3}}{4 a}, \quad i=1,2,3,
$$

the function $f_{2}$

$$
f_{2}=\sum_{1 \leq i, j \leq 3} e^{\theta_{i}+\eta_{j}+\alpha_{i j}}, \quad e^{\alpha_{i j}}=-\frac{1}{\left(k_{i}+\ell_{j}\right)^{2}}, \quad 1 \leq i, j \leq 3,
$$

the functions $F_{3}$ and $G_{3}$

$$
\begin{aligned}
& F_{3}=\sum_{\substack{1 \leq i, j, s \leq 3 \\
i<j}} A_{i j s} e^{\theta_{i}+\theta_{j}+\eta_{s}}, \quad A_{i j s}=-\frac{\left(k_{i}-k_{j}\right)^{2}}{\left(k_{i}+\ell_{s}\right)^{2}\left(k_{j}+\ell_{s}\right)^{2}}, \quad 1 \leq i, j, s \leq 3, \quad i<j, \\
& G_{3}=\sum_{\substack{1 \leq i, j, s \leq 3 \\
i<j}} B_{i j s} e^{\eta_{i}+\eta_{j}+\theta_{s}}, \quad B_{i j s}=-\frac{\left(\ell_{i}-\ell_{j}\right)^{2}}{\left(\ell_{i}+k_{s}\right)^{2}\left(\ell_{j}+k_{s}\right)^{2}}, \quad 1 \leq i, j, s \leq 3, \quad i<j,
\end{aligned}
$$

the function $f_{4}$

$$
f_{4}=\sum_{\substack{1 \leq i<j \leq 3 \\ 1 \leq p<r \leq 3}} M_{i j p r} e^{\theta_{i}+\theta_{j}+\eta_{p}+\eta_{r}}
$$

where

$$
M_{i j p r}=\frac{\left(k_{i}-k_{j}\right)^{2}\left(l_{p}-l_{r}\right)^{2}}{\left(k_{i}+l_{p}\right)^{2}\left(k_{i}+l_{r}\right)^{2}\left(k_{j}+l_{p}\right)^{2}\left(k_{j}+l_{r}\right)^{2}},
$$

for $1 \leq i<j \leq 3,1 \leq p<r \leq 3$, and the functions $F_{5}$ and $G_{5}$

$$
\begin{aligned}
& F_{5}=V_{12} e^{\theta_{1}+\theta_{2}+\theta_{3}+\eta_{1}+\eta_{2}}+V_{13} e^{\theta_{1}+\theta_{2}+\theta_{3}+\eta_{1}+\eta_{3}}+V_{23} e^{\theta_{1}+\theta_{2}+\theta_{3}+\eta_{2}+\eta_{3}}, \\
& G_{5}=W_{12} e^{\theta_{1}+\theta_{2}+\eta_{1}+\eta_{2}+\eta_{3}}+W_{13} e^{\theta_{1}+\theta_{2}+\eta_{1}+\eta_{2}+\eta_{3}}+W_{23} e^{\theta_{2}+\theta_{3}+\eta_{1}+\eta_{2}+\eta_{3}},
\end{aligned}
$$

where

$$
\begin{aligned}
& V_{i j}=\frac{S_{i j}}{4 a\left(\omega_{1}+\omega_{2}+\omega_{3}+m_{i}+m_{j}\right)+\left(k_{1}+k_{2}+k_{3}+\ell_{i}+\ell_{j}\right)^{3}}, \\
& W_{i j}=\frac{Q_{i j}}{4 a\left(\omega_{i}+\omega_{j}+m_{1}+m_{2}+m_{3}\right)+\left(k_{i}+k_{j}+\ell_{1}+\ell_{2}+\ell_{3}\right)^{3}},
\end{aligned}
$$

for $1 \leq i<j \leq 3$. Here $S_{i j}$ and $Q_{i j}$ are given in Appendix of Ref. [33]. We also obtain the function $f_{6}$ as

$$
f_{6}=H e^{\theta_{1}+\theta_{2}+\theta_{3}+\eta_{1}+\eta_{2}+\eta_{3}}
$$


where the coefficient $H$ is also given in Appendix of Ref. [33]. Let us also take $\varepsilon=1$. Hence three-soliton solution of the coupled mKdV system (1) and (2) is given with the pair $(q(t, x), r(t, x))$ where

$$
\begin{gathered}
q(t, x)=\frac{e^{\theta_{1}}+e^{\theta_{2}}+e^{\theta_{3}}+\sum_{\substack{1 \leq i, j, s \leq 3 \\
i<j}} A_{i j s} e^{\theta_{i}+\theta_{j}+\eta_{s}}+\sum_{\substack{1 \leq i, j \leq 3 \\
i<j}} V_{i j} e^{\theta_{1}+\theta_{2}+\theta_{3}+\eta_{i}+\eta_{j}}}{1+\sum_{1 \leq i, j \leq 3} e^{\theta_{i}+\eta_{j}+\alpha_{i j}}+\sum_{\substack{1 \leq i<j \leq 3 \\
1 \leq p<r \leq 3}} M_{i j p r} e^{\theta_{i}+\theta_{j}+\eta_{p}+\eta_{r}}+H e^{\theta_{1}+\theta_{2}+\theta_{3}+\eta_{1}+\eta_{2}+\eta_{3}}}, \\
r(t, x)=\frac{e^{\eta_{1}}+e^{\eta_{2}}+e^{\eta_{3}}+\sum_{\substack{1 \leq i, j, s \leq 3 \\
i<j}} B_{i j s} e^{\eta_{i}+\eta_{j}+\theta_{s}}+\sum_{\substack{1 \leq i, j \leq 3 \\
i<j}} W_{i j} e^{\theta_{i}+\theta_{j}+\eta_{1}+\eta_{2}+\eta_{3}}}{1+\sum_{1 \leq i, j \leq 3} e^{\theta_{i}+\eta_{j}+\alpha_{i j}}+\sum_{\substack{1 \leq i<j \leq 3 \\
1 \leq p<r \leq 3}} M_{i j p r} e^{\theta_{i}+\theta_{j}+\eta_{p}+\eta_{r}}+H e^{\theta_{1}+\theta_{2}+\theta_{3}+\eta_{1}+\eta_{2}+\eta_{3}}} .
\end{gathered}
$$

Having obtained the one-, two-, and three-soliton solutions of the mKdV system we now ready to obtain such soliton solutions of the nonlocal reductions of the $\mathrm{mKdV}$ system. Soliton solutions of the local reductions of the mKdV system can be found in [33]. Here in our solutions we focus on the domain $t \geq 0, x \in \mathbb{R}$.

\section{Nonlocal reductions of the MKdV system}

To find the soliton solutions of the nonlocal integrable equations which are obtained by consistent nonlocal reductions of an integrable system of equations we use the following three steps.

(i) Find consistent reduction formulas which reduce the integrable system equations to integrable nonlocal equations.

(ii) Find soliton solutions of the system equations by use of the Hirota direct method or by inverse scattering transform technique, or by use of Darboux Transformation.

(iii) Use the reduction formulas on the soliton solutions of the system equations to obtain the soliton solutions of the reduced nonlocal equations. By this way one obtains many different relations among the soliton parameters of the system equations.

In this section, using the above method, we will first use the reduction (7) given by Ablowitz and Musslimani [1] and $[7,8]$ and obtain soliton solutions for three different nonlocal cmKdV equations (9)-(11) with the condition

$$
\bar{a}=\varepsilon_{1} \varepsilon_{2} a
$$

satisfied. Secondly, we will deal with the reduction (12) and obtain one- and two-soliton solutions of the nonlocal mKdV equation (14).

\subsection{One-soliton solution for the nonlocal CMKdV equations: $\left(r=k \bar{q}\left(\varepsilon_{1} t, \varepsilon_{2} x\right)\right)$}

We have two types of soliton solutions of the reduced nonlocal equations. The main idea here is to use the one-soliton solutions (24) of the mKdV system equations (1)-(2) and then use the reduction formulas (7) and (12). By this procedure we obtain two types of soliton solutions of the reduced nonlocal equations.

\subsubsection{Type 1}

Firstly, we find the conditions on the parameters of one-soliton solution of the mKdV system to satisfy the constraint equation (7). Using this constraint equation we get

$$
\frac{e^{k_{2} x-\frac{k_{2}^{3}}{4 a} t+\delta_{2}}}{1+A e^{\left(k_{1}+k_{2}\right) x-\frac{\left(k_{1}^{3}+k_{2}^{3}\right)}{4 a} t+\delta_{1}+\delta_{2}}}=k \frac{e^{\bar{k}_{1} \varepsilon_{2} x-\frac{\bar{k}_{1}^{3}}{4 a} \varepsilon_{1} t+\bar{\delta}_{1}}}{1+\bar{A} e^{\left(\bar{k}_{1}+\bar{k}_{2}\right) \varepsilon_{2} x-\frac{\left.\bar{k}_{1}^{3}+\bar{k}_{2}^{3}\right)}{4 \bar{a}} \varepsilon_{1} t+\bar{\delta}_{1}+\bar{\delta}_{2}}} .
$$

This equation gives two different relations among the soliton parameters. One of the case (type-1) includes the following equalities that must be satisfied by the parameters:

$$
\begin{aligned}
& \text { i) } \left.k_{2}=\varepsilon_{2} \bar{k}_{1}, \quad \text { ii) } \frac{k_{2}^{3}}{4 a}=\frac{\bar{k}_{1}^{3}}{4 \bar{a}} \varepsilon_{1}, \quad \text { iii) } e^{\delta_{2}}=k e^{\bar{\delta}_{1}}, \quad \text { iv }\right) A=\bar{A}, \\
& \text { v) } \left.\left.\left(k_{1}+k_{2}\right)=\left(\bar{k}_{1}+\bar{k}_{2}\right) \varepsilon_{2}, \quad \text { vi }\right) \frac{\left(k_{1}^{3}+k_{2}^{3}\right)}{4 a}=\frac{\left(\bar{k}_{1}^{3}+\bar{k}_{2}^{3}\right)}{4 \bar{a}} \varepsilon_{1}, \quad \text { vii }\right) e^{\delta_{1}+\delta_{2}}=e^{\bar{\delta}_{1}+\bar{\delta}_{2}}
\end{aligned}
$$

If we use the conditions (51) and $i$ ) on the left hand side of the equality $i i)$, it is clear that this equality is satisfied directly since

$$
\frac{k_{2}^{3}}{4 a}=\frac{\varepsilon_{2} \bar{k}_{1}^{3}}{4 \varepsilon_{1} \varepsilon_{2} \bar{a}}=\frac{\bar{k}_{1}^{3}}{4 \bar{a}} \varepsilon_{1} .
$$

With the condition given in $i$ ) it is obvious that $i v$ ) is satisfied directly since

$$
-\frac{1}{\left(k_{1}+k_{2}\right)^{2}}=-\frac{1}{\left(\bar{k}_{2} \varepsilon_{2}+\bar{k}_{1} \varepsilon_{2}\right)^{2}}=-\frac{1}{\left(\bar{k}_{1}+\bar{k}_{2}\right)^{2}} \text {. }
$$


The condition $v$ ) is satisfied since

$$
\left(k_{1}+k_{2}\right)=\left(\bar{k}_{2} \varepsilon_{2}+\bar{k}_{1} \varepsilon_{2}\right)=\left(\bar{k}_{1}+\bar{k}_{2}\right) \varepsilon_{2}
$$

by the condition $k_{2}=\varepsilon_{2} \bar{k}_{1}$ or equivalently $k_{1}=\varepsilon_{2} \bar{k}_{2}$. By the same manner vi) is already true since

$$
\frac{\left(k_{1}^{3}+k_{2}^{3}\right)}{4 a}=\frac{\left(\bar{k}_{2}^{3}+\bar{k}_{1}^{3}\right)}{4 \varepsilon_{1} \varepsilon_{2} \bar{a}}=\frac{\left(\bar{k}_{1}^{3}+\bar{k}_{2}^{3}\right)}{4 \bar{a}} \varepsilon_{1} .
$$

Finally, consider the relation $e^{\delta_{2}}=k e^{\bar{\delta}_{1}}$ or $e^{\bar{\delta}_{2}}=k e^{\delta_{1}}$ given in vii). Since $k$ is a real constant we have $e^{\delta_{1}+\delta_{2}}=k e^{\delta_{1}} e^{\bar{\delta}_{1}}$ and $e^{\bar{\delta}_{1}+\bar{\delta}_{2}}=k e^{\bar{\delta}_{1}} e^{\delta_{1}}$ that yield the equality $e^{\delta_{1}+\delta_{2}}=e^{\bar{\delta}_{1}+\bar{\delta}_{2}}$.

Thus the parameters of one-soliton solution of the Eq. (8) must have the following properties:

$$
\text { 1) } \bar{a}=\varepsilon_{1} \varepsilon_{2} a, \text { 2) } k_{2}=\varepsilon_{2} \bar{k}_{1}, \quad \text { 3) } e^{\delta_{2}}=k e^{\bar{\delta}_{1}} \text {. }
$$

The case $\left(\varepsilon_{1}, \varepsilon_{2}\right)=(1,1)$ gives local equation. For particular choice of the parameters let us check the solutions of the nonlocal reductions of the mKdV system for $\left(\varepsilon_{1}, \varepsilon_{2}\right)=\{(-1,1),(1,-1),(-1,-1)\}$.

3.1.1.1. Case a. (T-symmetric). $r=k \bar{q}(-t, x)$. This case gives $\bar{a}=-a, k_{2}=\bar{k}_{1}$, and

$$
a q_{t}(t, x)=-\frac{1}{4} q_{x x x}(t, x)+\frac{3}{2} k \bar{q}(-t, x) q(t, x) q_{x}(t, x)
$$

with $e^{\delta_{2}}=k e^{\bar{\delta}_{1}}$. Since $\bar{a}=-a$, $a$ is pure imaginary say $a=i b$, for nonzero $b \in \mathbb{R}$. Let $k_{1}=\alpha+i \beta$ so $k_{2}=\alpha-i \beta$ for $\alpha, \beta \in \mathbb{R}$, $\alpha \neq 0$. Then the solution of (55) becomes

$$
q(t, x)=\frac{e^{(\alpha+i \beta) x+\frac{\left(\beta^{3}-3 \alpha^{2} \beta\right)+i\left(\alpha^{3}-3 \alpha \beta^{2}\right)}{4 b} t+\delta_{1}}}{1-\frac{k}{4 \alpha^{2}} e^{2 \alpha x+i \frac{\alpha^{3}-3 \alpha \beta^{2}}{2 b} t+\delta_{1}+\bar{\delta}_{1}}} .
$$

This solution is also given in [28]. The corresponding function $|q(t, x)|^{2}$ is

$$
|q(t, x)|^{2}=\frac{e^{2 \alpha x+\frac{\left(\beta^{3}-3 \alpha^{2} \beta\right)}{2 b} t+\delta_{1}+\bar{\delta}_{1}}}{\left[\frac{k}{4 \alpha^{2}} e^{2 \alpha x+\delta_{1}+\bar{\delta}_{1}}-\cos \left(\frac{\left(\alpha^{3}-3 \alpha \beta^{2}\right)}{2 b} t\right)\right]^{2}+\sin ^{2}\left(\frac{\left(\alpha^{3}-3 \alpha \beta^{2}\right)}{2 b} t\right)} .
$$

When $\alpha^{3}-3 \alpha \beta^{2} \neq 0$ and $t=\frac{2 n b \pi}{\alpha^{3}-3 \alpha \beta^{2}}, \frac{k}{4 \alpha^{2}} e^{2 \alpha x+\delta_{1}+\bar{\delta}_{1}}-(-1)^{n}=0$ where $n$ is an integer, for both focusing and defocusing cases, the solution is singular. When $\alpha^{3}-3 \alpha \beta^{2}=0$ the solution for focusing case is non-singular. When $\alpha=0$ the solution is exponentially growing for $\frac{\beta^{3}}{b}>0$ and exponentially decaying for $\frac{\beta^{3}}{b}<0$. Now for particular choices of the parameters satisfying the conditions (54) we give an example of a solution of the Eq. (55) and present the graph of the solution.

Example 1. For the parameters $\left(k_{1}, k_{2}, e^{\delta_{1}}, e^{\delta_{2}}, k, a\right)=(2 \sqrt{3}+2 i, 2 \sqrt{3}-2 i, 1+i,-1+i,-1,10 i)$ we obtain the non-singular solution of (55) as

$$
q(t, x)=\frac{24(1+i) e^{(2 \sqrt{3}+2 i) x-\frac{8}{5} t}}{24+e^{4 \sqrt{3} x}},
$$

so the function $|q(t, x)|^{2}$ is

$$
|q(t, x)|^{2}=12 e^{-\frac{16}{5} t} \operatorname{sech}^{2}(2 \sqrt{3} x+\delta),
$$

where $\delta=-\frac{1}{2} \ln (24)$. The solution is an asymptotically decaying solution for $t>0$. The graph of (59) is given in Fig. 1 .

3.1.1.2. Case $b$. (S-symmetric): $r=k \bar{q}(t,-x)$. In this case we have $\bar{a}=-a, k_{2}=-\bar{k}_{1}$, and

$$
a q_{t}(t, x)=-\frac{1}{4} q_{x x x}(t, x)+\frac{3}{2} k \bar{q}(t,-x) q(t, x) q_{x}(t, x)
$$

with $e^{\delta_{2}}=k e^{\bar{\delta}_{1}}$. Since $\bar{a}=-a$, it is pure imaginary, say $a=i b$ for nonzero $b \in \mathbb{R}$. Let also $k_{1}=\alpha+i \beta$ and so $k_{2}=-\alpha+i \beta$ for $\alpha, \beta \in \mathbb{R}, \beta \neq 0$. Then the solution of (60) becomes

$$
q(t, x)=\frac{e^{(\alpha+i \beta) x+\frac{\left(\beta^{3}-3 \alpha^{2} \beta\right)+i\left(\alpha^{3}-3 \alpha \beta^{2}\right)}{4 b} t+\delta_{1}}}{1+\frac{k}{4 \beta^{2}} e^{2 i \beta x+i \frac{\alpha^{3}-3 \alpha \beta^{2}}{2 b} t+\delta_{1}+\bar{\delta}_{1}}},
$$

and so the function $|q(t, x)|^{2}$ is

$$
|q(t, x)|^{2}=\frac{e^{2 \alpha x+\frac{\left(\beta^{3}-3 \alpha^{2} \beta\right)}{2 b} t+\delta_{1}+\bar{\delta}_{1}}}{\left[\frac{k}{4 \beta^{2}} e^{\frac{\left(\beta^{3}-3 \alpha^{2} \beta\right)}{2 b} t+\delta_{1}+\bar{\delta}_{1}}+\cos (2 \beta x)\right]^{2}+\sin ^{2}(2 \beta x)} .
$$




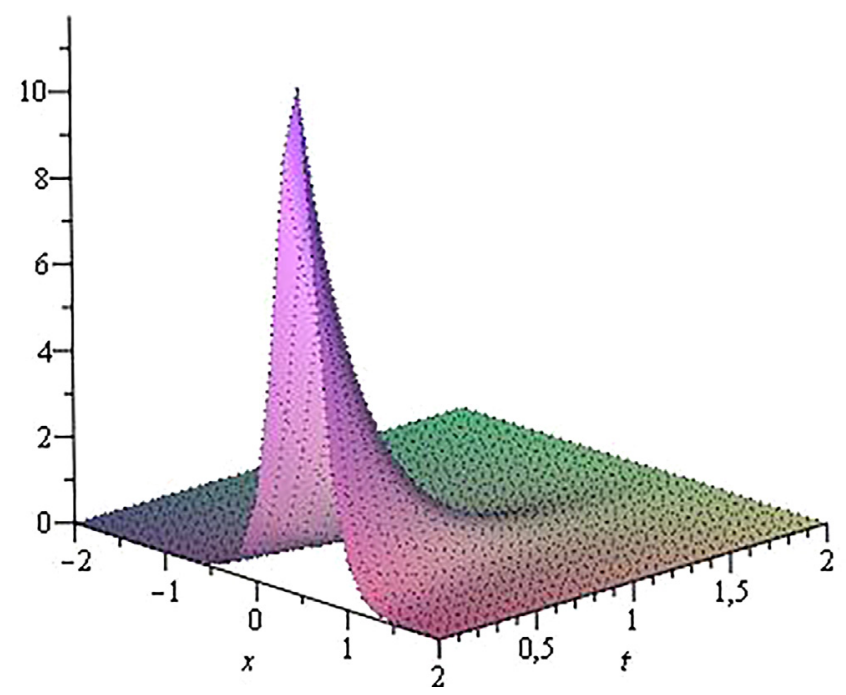

Fig. 1. A non-singular localized wave for (57) with the parameters $k_{1}=\alpha+i \beta=2 \sqrt{3}+2 i, e^{\delta_{1}}=1+i, k=-1, a=i b=10 i$.

For $x=\frac{n \pi}{2 \beta}$ and $\frac{k}{4 \beta^{2}} e^{\frac{\left(\beta^{3}-3 \alpha^{2} \beta\right)}{2 b} t+\delta_{1}+\bar{\delta}_{1}}+(-1)^{n}=0$, where $n$ is an integer, the solution is unbounded but for $\beta^{2}=3 \alpha^{2}$ and $\frac{k}{4 \beta^{2}} e^{\delta_{1}+\bar{\delta}_{1}}+(-1)^{n} \neq 0$ we have a periodical solution. For $\alpha=0$, the solution (62) becomes

$$
|q(t, x)|^{2}=\frac{e^{\delta_{1}+\bar{\delta}_{1}}}{\gamma\left[\sigma_{k} \cosh \left(\frac{\beta^{3}}{2 b} t+\ln \left(\frac{|\gamma|}{2}\right)\right)+\cos (2 \beta x)\right]},
$$

where $\gamma=\frac{k}{2 \beta^{2}} e^{\delta_{1}+\bar{\delta}_{1}}, \sigma_{k}=1$ if $k>0$, and $\sigma_{k}=-1$ if $k<0$. This solution is non-singular for $|\gamma|>2, \frac{\beta^{3}}{b}>0$ and $|\gamma|<2$, $\frac{\beta^{3}}{b}<0$. Let us give the following example for a solution of $(60)$.

Example 2. For the parameters $\left(k_{1}, k_{2}, e^{\delta_{1}}, e^{\delta_{2}}, k, a\right)=\left(\frac{i}{4}, \frac{i}{4}, 1+i, 1-i, 1, \frac{i}{2}\right)$, we get the solution $q(t, x)$ of $(60)$ as

$$
q(t, x)=\frac{(1+i) e^{\frac{1}{4} i x+\frac{1}{128} t}}{1+8 e^{\frac{1}{2} i x+\frac{1}{164} t}} .
$$

Hence the function $|q(t, x)|^{2}$ is

$$
|q(t, x)|^{2}=\frac{1}{8\left[\cosh \left(\frac{t}{64}+\ln 8\right)+\cos \left(\frac{1}{2} x\right)\right]} .
$$

This is a periodic and bounded solution for $x \in \mathbb{R}$ and decreases with respect to $t$ for all real $t \neq-192 \ln 2$. The graph of the solution (65) is given in Fig. 2.

3.1.1.3. Case c. (ST-symmetric): $r=k \bar{q}(-t,-x)$. For this case we have $\bar{a}=a, k_{2}=-\bar{k}_{1}$, and

$$
a q_{t}(t, x)=-\frac{1}{4} q_{x x x}(t, x)+\frac{3}{2} k \bar{q}(-t,-x) q(t, x) q_{x}(t, x),
$$

with $e^{\delta_{2}}=k e^{\bar{\delta}_{1}}$. Let $k_{1}=\alpha+i \beta$ and so $k_{2}=-\alpha+i \beta$ for $\alpha, \beta \in \mathbb{R}, \beta \neq 0$. Then the solution $q(t, x)$ of (66) becomes

$$
q(t, x)=\frac{e^{(\alpha+i \beta) x-\frac{\left(\alpha^{3}-3 \alpha \beta^{2}\right)+i\left(3 \alpha^{2} \beta-\beta^{3}\right)}{4 a} t+\delta_{1}}}{1+\frac{k}{4 \beta^{2}} e^{2 i \beta x-i \frac{\left(6 \alpha^{2} \beta-2 \beta^{3}\right)}{4 a} t+\delta_{1}+\bar{\delta}_{1}}} .
$$

Then we obtain the function $|q(t, x)|^{2}$ as

$$
|q(t, x)|^{2}=\frac{e^{2 \alpha x+\frac{\left(3 \alpha \beta^{2}-\alpha^{3}\right)}{2 a} t+\delta_{1}+\bar{\delta}_{1}}}{\left[\frac{k}{4 \beta^{2}} e^{\delta_{1}+\bar{\delta}_{1}}+\cos \left(2 \beta x+\frac{\left(\beta^{3}-3 \alpha^{2} \beta\right)}{2 a} t\right)\right]^{2}+\sin ^{2}\left(2 \beta x+\frac{\left(\beta^{3}-3 \alpha^{2} \beta\right)}{2 a} t\right)} .
$$

Let $\theta=2 \alpha x+\frac{\left(3 \alpha \beta^{2}-\alpha^{3}\right)}{2 a} t+\delta_{1}+\bar{\delta}_{1}$ and $\phi=2 \beta x+\frac{\left(\beta^{3}-3 \alpha^{2} \beta\right)}{2 a} t$. In this case the solution (68) can be written as

$$
|q(t, x)|^{2}=\frac{e^{\theta}}{1+\frac{\mu^{2}}{4}+\mu \cos \phi}=\frac{e^{\theta}}{\mu\left[\left(\frac{1}{\mu}+\frac{\mu}{4}\right)+\cos \phi\right]},
$$




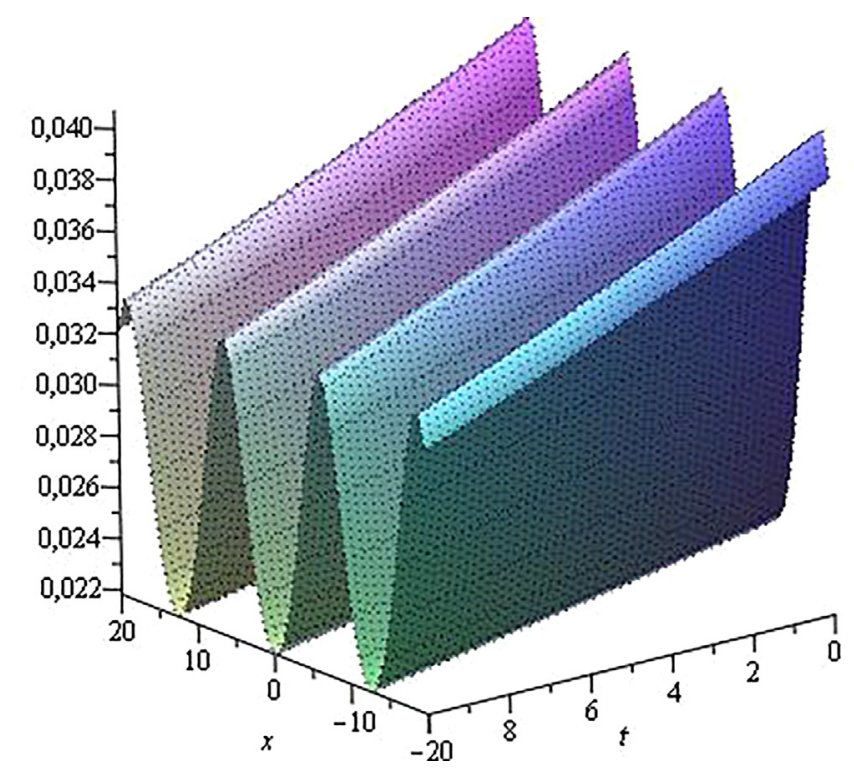

Fig. 2. A periodical decaying wave for (62) with the parameters $k_{1}=\alpha+i \beta=\frac{i}{4}, e^{\delta_{1}}=1+i, k=1, a=i b=\frac{i}{2}$.

where $\mu=\frac{k}{2 \beta^{2}} e^{\delta_{1}+\bar{\delta}_{1}}$. This solution is non-singular for all $\mu$ except $\mu= \pm 2$. Note that if we take $k=1, a=\frac{1}{4}, \alpha=2 b$, $\beta=2 a, e^{\delta_{1}}=-4 a i$, in our solution (67) which corresponds to the case for $\mu=2$ then the solution (67) reduces to one of the solutions given in [25]. For particular choice of parameters let us present the following examples for this case.

Example 3. When $\alpha=0$, then $\theta$ becomes constant and the solution (69) is finite and periodical. As an example choose the parameters as $\left(k_{1}, k_{2}, e^{\delta_{1}}, e^{\delta_{2}}, k, a\right)=\left(\frac{i}{4}, \frac{i}{4}, 1+i, 1-i, 1,2\right)$. We have the solution of (66) as

$$
q(t, x)=\frac{(1+i) e^{\frac{1}{4} i x+\frac{1}{512} i t}}{1+8 e^{\frac{1}{2} i x+\frac{1}{256} i t}} .
$$

Then we obtain the function $|q(t, x)|^{2}$,

$$
|q(t, x)|^{2}=\frac{2}{65+16 \cos \left(\frac{1}{2} x+\frac{1}{256} t\right)},
$$

which is a periodical solution. The graph of (71) is given in Fig. 3.

Example 4. For the parameters $\left(k_{1}, k_{2}, e^{\delta_{1}}, e^{\delta_{2}}, k, a\right)=\left(\frac{4}{10}+\frac{42}{100} i,-\frac{4}{10}+\frac{42}{100} i, 1,1,1,-4\right)$, we have the solution of (66),

$$
q(t, x)=\frac{e^{\left(\frac{2}{5}+\frac{21}{50} i\right) x+\left(-\frac{923}{100000}+\frac{15939}{2000000} i\right) t}}{1+\frac{625}{441} e^{\frac{21}{25} i x+\frac{15939}{100000} i t}}
$$

Therefore the function $|q(t, x)|^{2}$ is found as

$$
|q(t, x)|^{2}=\frac{194481 e^{\frac{4}{5} x-\frac{923}{50000} t}}{2\left(292553+275625 \cos \left(\frac{21}{25} x+\frac{15939}{1000000} t\right)\right)} .
$$

The above solution is an asymptotically growing wave solution as $x \rightarrow \infty$.

\subsubsection{Type 2}

From the Eq. (52), we obtain a second set of relations among the soliton parameters by performing the cross multiplication in (52) as

$$
\begin{aligned}
& e^{k_{2} x-\frac{k_{2}^{3}}{4 a} t+\delta_{2}}+\bar{A} e^{\left[\left(\bar{k}_{1}+\bar{k}_{2}\right) \varepsilon_{2}+k_{2}\right] x-\left[\frac{\left.\bar{k}_{1}^{3}+\bar{k}_{2}^{3}\right) \varepsilon_{1}}{4 a}+\frac{k_{2}^{3}}{4 a}\right] t+\bar{\delta}_{1}+\bar{\delta}_{2}+\delta_{2}} \\
& =k e^{\bar{k}_{1} \varepsilon_{2} x-\frac{\bar{k}_{1}^{3}}{4 a} \varepsilon_{1} t+\bar{\delta}_{1}}+A k e^{\left[\left(k_{1}+k_{2}\right)+\bar{k}_{1} \varepsilon_{2}\right] x-\left[\frac{\left(k_{1}^{3}+k_{2}^{3}\right)}{4 a}+\frac{\bar{k}_{1}^{3}}{4 a} \varepsilon_{1}\right] t+\delta_{1}+\delta_{2}+\bar{\delta}_{1}} .
\end{aligned}
$$

Here in addition to the Type 1 conditions, we find the following new conditions in Type 2:

$$
\text { i) } k_{2}=k_{1}+k_{2}+\bar{k}_{1} \varepsilon_{2}, \quad \text { ii) } \frac{k_{2}^{3}}{4 a}=\frac{k_{1}^{3}+k_{2}^{3}}{4 a}+\frac{\bar{k}_{1}^{3}}{4 \bar{a}} \varepsilon_{1}, \quad \text { iii) } e^{\delta_{2}}=A k e^{\delta_{1}+\delta_{2}+\bar{\delta}_{1}},
$$




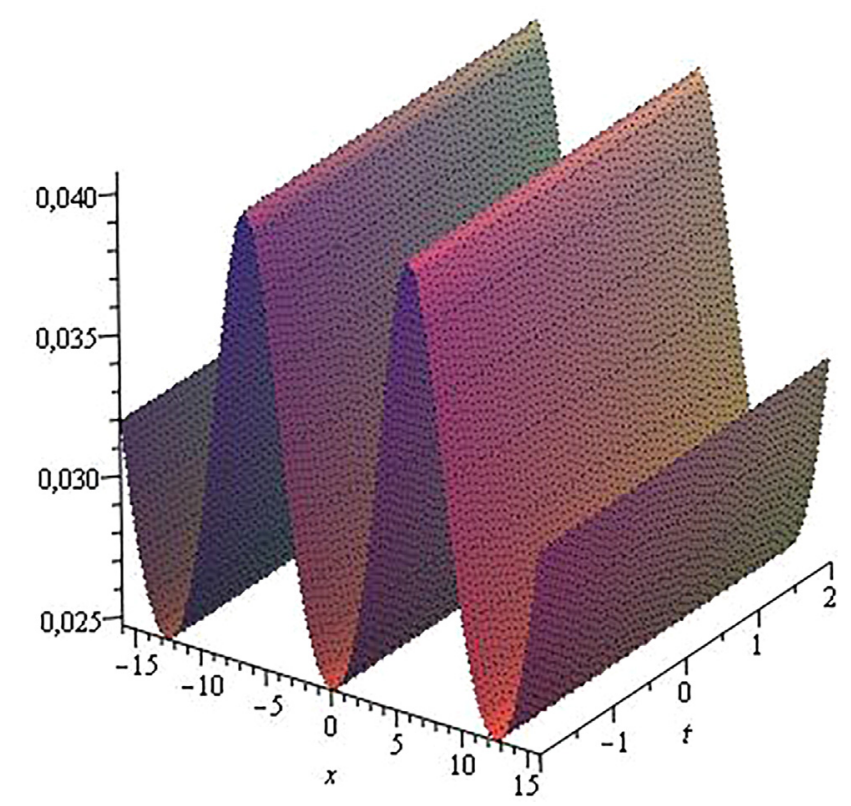

Fig. 3. A periodical wave for (68) with the parameters $k_{1}=\alpha+i \beta=\frac{i}{4}, e^{\delta_{1}}=1+i, k=1, a=2$.
iv) $\bar{k}_{1} \varepsilon_{2}+\bar{k}_{2} \varepsilon_{2}+k_{2}=\bar{k}_{1} \varepsilon_{2}$,
v) $\frac{\bar{k}_{1}^{3}+\bar{k}_{2}^{3}}{4 \bar{a}} \varepsilon_{1}+\frac{k_{2}^{3}}{4 a}=\frac{\bar{k}_{1}^{3}}{4 \bar{a}} \varepsilon_{1}$,
vi) $\bar{A} e^{\bar{\delta}_{1}+\bar{\delta}_{2}+\delta_{2}}=k e^{\bar{\delta}_{1}}$

Clearly, these conditions are simplified as
1) $\bar{a}=\varepsilon_{1} \varepsilon_{2} a$,
2) $k_{1}=-\bar{k}_{1} \varepsilon_{2}$,
3) $k_{2}=-\bar{k}_{2} \varepsilon_{2}$,
4) $A k e^{\delta_{1}+\bar{\delta}_{1}}=1$,
5) $A e^{\delta_{2}+\bar{\delta}_{2}}=k$.

Let us present some particular examples satisfying the above constraints.

3.1.2.1. Case a. (T-symmetric): $r=k \bar{q}(-t, x)$. Here the parameters satisfy $\bar{a}=-a, k_{1}=-\bar{k}_{1}, k_{2}=-\bar{k}_{2}$ with $A k e^{\delta_{1}+\bar{\delta}_{1}}=1$, $A e^{\delta_{2}+\bar{\delta}_{2}}=k$. In this case the parameters $a, k_{1}$, and $k_{2}$ are pure imaginary, say $a=i \alpha, k_{1}=i \beta$, and $k_{2}=i \gamma$ for $\alpha, \beta, \gamma \in \mathbb{R}$. Therefore we have $e^{\delta_{1}+\bar{\delta}_{1}}=\frac{(\beta+\gamma)^{2}}{k}$ and $e^{\delta_{2}+\bar{\delta}_{2}}=k(\beta+\gamma)^{2}$. Let also $e^{\delta_{1}}=a_{1}+i b_{1}$ and $e^{\delta_{2}}=a_{2}+i b_{2}$ for $a_{1}, b_{1}, a_{2}, b_{2} \in \mathbb{R}$. Then one-soliton solution becomes

$$
q(t, x)=\frac{e^{i \beta x+\frac{\beta^{3}}{4 \alpha} t}\left(a_{1}+i b_{1}\right)}{1+\frac{1}{(\beta+\gamma)^{2}} e^{i(\beta+\gamma) x+\frac{\left(\beta^{3}+\gamma^{3}\right)}{4 \alpha} t}\left(a_{1}+i b_{1}\right)\left(a_{2}+i b_{2}\right)} .
$$

Hence the function $|q(t, x)|^{2}$ is

$$
|q(t, x)|^{2}=\frac{e^{\frac{\beta^{3}}{2 \alpha} t}\left(a_{1}^{2}+b_{1}^{2}\right)}{1+\frac{2}{(\beta+\gamma)^{2}} e^{\frac{\left(\beta^{3}+\gamma^{3}\right)}{4 \alpha} t}\left[\left(a_{1} a_{2}-b_{1} b_{2}\right) \cos ((\beta+\gamma) x)-\left(a_{1} b_{2}+a_{2} b_{1}\right) \sin ((\beta+\gamma) x)\right]+e^{\frac{\left(\beta^{3}+\gamma^{3}\right)}{2 \alpha} t},},
$$

where $a_{1}^{2}+b_{1}^{2}=\frac{(\beta+\gamma)^{2}}{k}$ and $a_{2}^{2}+b_{2}^{2}=k(\beta+\gamma)^{2}, \beta \neq-\gamma$. Let $a_{1} a_{2}-b_{1} b_{2}=B \cos \omega_{0}, a_{1} b_{2}+a_{2} b_{1}=B \sin \omega_{0}$ for $B>0$. Hence

$$
B^{2}=\left(a_{1}^{2}+b_{1}^{2}\right)\left(a_{2}^{2}+b_{2}^{2}\right)=(\beta+\gamma)^{4},
$$

yielding $B=(\beta+\gamma)^{2}$. In this case, the solution (77) can be written as

$$
|q(t, x)|^{2}=\frac{e^{\frac{\left(\beta^{3}-\gamma^{3}\right)}{4 \alpha} t}\left(a_{1}^{2}+b_{1}^{2}\right)}{2\left[\cosh \left(\frac{\left(\beta^{3}+\gamma^{3}\right)}{4 \alpha} t\right)+\cos \theta\right]},
$$

where $\theta=(\beta+\gamma) x+\omega_{0}$. This solution is singular only at $t=0, \theta=(2 n+1) \pi$ for $n$ integer.

Example 5. Choose $\left(k_{1}, k_{2}, e^{\delta_{1}}, e^{\delta_{2}}, k, a\right)=\left(i,-\frac{i}{2}, 1, \frac{1}{4}, \frac{1}{4}, 2 i\right)$. Hence the solution becomes

$$
q(t, x)=\frac{e^{i x+\frac{1}{8} t}}{1+e^{\frac{1}{2} i x+\frac{7}{64} t}},
$$


so we obtain the function $|q(t, x)|^{2}$ as

$$
|q(t, x)|^{2}=\frac{e^{\frac{9}{64} t}}{2\left[\cosh \left(\frac{7}{64} t\right)+\cos \left(\frac{1}{2} x\right)\right]}
$$

The above function has singularity at $(t, x)=(0,2(2 n+1) \pi), n$ integer.

3.1.2.2. Case $b$. (S-symmetric): $r=k \bar{q}(t,-\chi)$. For this case we have $\bar{a}=-a, k_{1}=\bar{k}_{1}, k_{2}=\bar{k}_{2}$ with $A k e^{\delta_{1}+\bar{\delta}_{1}}=1, A e^{\delta_{2}+\bar{\delta}_{2}}=k$. Thus $a$ is pure imaginary, say $a=i \alpha, \alpha \in \mathbb{R}$ and the parameters $k_{1}$ and $k_{2}$ are real. Let $e^{\delta_{1}}=a_{1}+i b_{1}$ and $e^{\delta_{2}}=a_{2}+i b_{2}$ for $a_{1}, b_{1}, a_{2}, b_{2} \in \mathbb{R}$. Hence one-soliton solution becomes

$$
q(t, x)=\frac{e^{k_{1} x+i \frac{k_{1}^{3}}{4 \alpha} t}\left(a_{1}+i b_{1}\right)}{1-\frac{1}{\left(k_{1}+k_{2}\right)^{2}} e^{\left(k_{1}+k_{2}\right) x+i \frac{\left(k_{1}^{3}+k_{2}^{3}\right)}{4 \alpha} t}\left(a_{1}+i b_{1}\right)\left(a_{2}+i b_{2}\right)} .
$$

Therefore we obtain the function $|q(t, x)|^{2}$ as

$$
|q(t, x)|^{2}=\frac{e^{2 k_{1} x}\left(a_{1}^{2}+b_{1}^{2}\right)}{1-\frac{2 e^{\left(k_{1}+k_{2}\right) x}}{\left(k_{1}+k_{2}\right)^{2}}\left[\cos \left(\frac{\left(k_{1}^{3}+k_{2}^{3}\right)}{4 \alpha} t\right)\left(a_{1} a_{2}-b_{1} b_{2}\right)-\sin \left(\frac{\left(k_{1}^{3}+k_{2}^{3}\right)}{4 \alpha} t\right)\left(a_{1} b_{2}+b_{1} a_{2}\right)\right]+e^{2\left(k_{1}+k_{2}\right) x},}
$$

where $a_{1}^{2}+b_{1}^{2}=-\frac{\left(k_{1}+k_{2}\right)^{2}}{k}$ and $a_{2}^{2}+b_{2}^{2}=-k\left(k_{1}+k_{2}\right)^{2}, k_{1} \neq-k_{2}$. Let $a_{1} a_{2}-b_{1} b_{2}=-B \cos \omega_{0}, a_{1} b_{2}+b_{1} a_{2}=B \sin \omega_{0}$ for $B>0$. Therefore $B=\left(k_{1}+k_{2}\right)^{2}$. The solution (83) can be expressed as

$$
|q(t, x)|^{2}=\frac{e^{\left(k_{1}-k_{2}\right) x}\left(a_{1}^{2}+b_{1}^{2}\right)}{2\left[\cosh \left(\left(k_{1}+k_{2}\right) x\right)+\cos \theta\right]},
$$

where $\theta=\frac{\left(k_{1}^{3}+k_{2}^{3}\right)}{4} t-\omega_{0}$. This solution has singularity at $x=0, \theta=(2 n+1) \pi$ for $n$ integer.

Example 6. Take $\left(k_{1}, k_{2}, e^{\delta_{1}}, e^{\delta_{2}}, k, a\right)=(1,1,-2,2,-1,2 i)$. So the solution $q(t, x)$ becomes

$$
q(t, x)=\frac{-2 e^{x+\frac{1}{8} i t}}{1+e^{2 x+\frac{1}{4} i t}},
$$

and the function $|q(t, x)|^{2}$ is

$$
|q(t, x)|^{2}=\frac{2}{\cosh (2 x)+\cos \left(\frac{1}{4} t\right)}
$$

The above function has singularity at $(t, x)=(4(2 n+1) \pi, 0), n$ integer

3.1.2.3. Case c. (ST-symmetric): $r=k \bar{q}(-t,-x)$. Here the parameters satisfy $\bar{a}=a, k_{1}=\bar{k}_{1}, k_{2}=\bar{k}_{2}$ with $A k e^{\delta_{1}+\bar{\delta}_{1}}=1$, $A e^{\delta_{2}+\bar{\delta}_{2}}=k$. Therefore $a, k_{1}$, and $k_{2}$ are real. Let also $e^{\delta_{1}}=a_{1}+i b_{1}$ and $e^{\delta_{2}}=a_{2}+i b_{2}$ for $a_{1}, b_{1}, a_{2}, b_{2} \in \mathbb{R}$. Then one-soliton solution becomes

$$
q(t, x)=\frac{e^{k_{1} x-\frac{k_{1}^{3}}{4 a} t}\left(a_{1}+i b_{1}\right)}{1-\frac{1}{\left(k_{1}+k_{2}\right)^{2}} e^{\left(k_{1}+k_{2}\right) x-\frac{\left(k_{1}^{3}+k_{2}^{3}\right)}{4 a} t}\left(a_{1}+i b_{1}\right)\left(a_{2}+i b_{2}\right)} .
$$

Hence we have the function $|q(t, x)|^{2}$ as

$$
|q(t, x)|^{2}=\frac{e^{2 k_{1} x-\frac{k_{1}^{3}}{2 a} t}\left(a_{1}^{2}+b_{1}^{2}\right)}{1-\frac{2\left(a_{1} a_{2}-b_{1} b_{2}\right)}{\left(k_{1}+k_{2}\right)^{2}} e^{\left(k_{1}+k_{2}\right) x-\frac{\left(k_{1}^{3}+k_{2}^{3}\right)}{4 a} t}+e^{2\left(k_{1}+k_{2}\right) x-\frac{\left(k_{1}^{3}+k_{2}^{3}\right)}{2 a} t},}
$$

where $a_{1}^{2}+b_{1}^{2}=-\frac{\left(k_{1}+k_{2}\right)^{2}}{k}$ and $a_{2}^{2}+b_{2}^{2}=-k\left(k_{1}+k_{2}\right)^{2}, k_{1} \neq-k_{2}$. If we let $\theta=\left(k_{1}+k_{2}\right) x-\frac{\left(k_{1}^{3}+k_{2}^{3}\right)}{4 a} t, \phi=2 k_{1} x-\frac{k_{1}^{3}}{2 a} t$, and $\gamma=$ $\frac{\left(a_{1} a_{2}-b_{1} b_{2}\right)}{\left(k_{1}+k_{2}\right)^{2}}$, then the solution (88) becomes

$$
|q(t, x)|^{2}=\frac{e^{\phi}}{1-2 \gamma e^{\theta}+e^{2 \theta}} .
$$

The above function has singularity when the function $f(\theta)=e^{2 \theta}-2 \gamma e^{\theta}+1$ vanishes. It becomes zero when $e^{\theta}=\gamma \pm$ $\sqrt{\gamma^{2}-1}$. Hence for $\gamma<1$ and $k_{2}>k_{1}$ the solution is non-singular and bounded.

Note that if we let $k=-1, a=\frac{1}{4}, k_{1}=-2 \bar{\eta}, k_{2}=-2 \eta, e^{\delta_{1}}=-2(\eta+\bar{\eta}) e^{i \bar{\theta}}$, and $e^{\delta_{2}}=2(\eta+\bar{\eta}) e^{i \theta}$, then one-soliton solution that we obtain here turns to be the same solution given by Ablowitz and Musslimani [8]. 


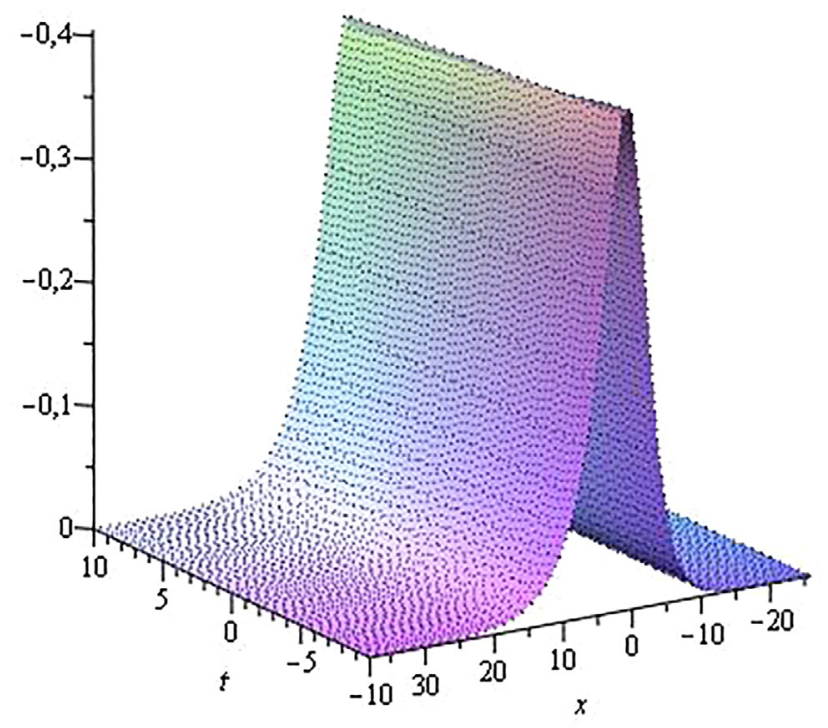

Fig. 4. Asymptotically decaying soliton for (88) with the parameters $k_{1}=\frac{1}{2}, k_{2}=\frac{1}{4}, e^{\delta_{1}}=a_{1}+i b_{1}=-\frac{3}{4}, e^{\delta_{2}}=a_{2}+i b_{2}=\frac{3}{4}, k=-1, a=2$.

Example 7. Consider the following set of the parameters: $\left(k_{1}, k_{2}, e^{\delta_{1}}, e^{\delta_{2}}, k, a\right)=\left(\frac{1}{2}, \frac{1}{4},-\frac{3}{4}, \frac{3}{4},-1,2\right)$. We obtain the following asymptotically decaying soliton

$$
q(t, x)=\frac{-3 e^{\frac{1}{2} x-\frac{1}{64} t}}{4\left(1+e^{\frac{3}{4} x-\frac{9}{512} t}\right)} .
$$

The graph of this function is given in Fig. 4 .

Example 8. For the parameters $\left(k_{1}, k_{2}, e^{\delta_{1}}, e^{\delta_{2}}, k, a\right)=(0,1, i, 1,-1,2)$ we have the solution

$$
q(t, x)=\frac{i}{1-i e^{x-\frac{1}{8} t}},
$$

and so

$$
|q(t, x)|^{2}=\frac{1}{1+e^{2 x-\frac{1}{4} t}} .
$$

This solution represents a kink-type wave and its graph is given in Fig. 5.

3.2. Two-soliton solution for the nonlocal CMKdV equations: $\left(r=k \bar{q}\left(\varepsilon_{1} t, \varepsilon_{2} x\right)\right)$

We first obtain the conditions on the parameters of two-soliton solution of the mKdV system to satisfy (7). Here the function $r(t, x)$ is given in (35) and $k \bar{q}\left(\varepsilon_{1} t, \varepsilon_{2} x\right)$ is

$$
k \bar{q}\left(\varepsilon_{1} t, \varepsilon_{2} x\right)=k \frac{e^{\bar{\theta}_{1}}+e^{\bar{\theta}_{2}}+\bar{\gamma}_{1} e^{\bar{\theta}_{1}+\bar{\theta}_{2}+\bar{\eta}_{1}}+\bar{\gamma}_{2} e^{\bar{\theta}_{1}+\bar{\theta}_{2}+\bar{\eta}_{2}}}{1+e^{\bar{\theta}_{1}+\bar{\eta}_{1}+\bar{\alpha}_{11}}+e^{\bar{\theta}_{1}+\bar{\eta}_{2}+\bar{\alpha}_{12}}+e^{\bar{\theta}_{2}+\bar{\eta}_{1}+\bar{\alpha}_{21}}+e^{\bar{\theta}_{2}+\bar{\eta}_{2}+\bar{\alpha}_{22}}+\bar{M} e^{\bar{\theta}_{1}+\bar{\theta}_{2}+\bar{\eta}_{1}+\bar{\eta}_{2}}},
$$

where

$$
\begin{aligned}
& \bar{\theta}_{i}=\varepsilon_{2} \bar{k}_{i} x-\varepsilon_{1} \frac{\bar{k}_{i}^{3}}{4 \bar{a}} t+\bar{\delta}_{i}, i=1,2, \\
& \bar{\eta}_{i}=\varepsilon_{2} \bar{\ell}_{i} x-\varepsilon_{1} \frac{\bar{\ell}_{i}^{3}}{4 \bar{a}} t+\bar{\alpha}_{i}, i=1,2 .
\end{aligned}
$$

We get the following conditions that must be satisfied:

$$
\begin{aligned}
& \text { i) } e^{\eta_{i}}=k e^{\bar{\theta}_{i}}, i=1,2, \quad \text { ii) } e^{\theta_{1}+\eta_{1}+\eta_{2}}=k e^{\bar{\theta}_{1}+\bar{\theta}_{2}+\bar{\eta}_{1}}, \quad \text { iii) } e^{\theta_{2}+\eta_{1}+\eta_{2}}=k e^{\bar{\theta}_{1}+\bar{\theta}_{2}+\bar{\eta}_{2}}, \\
& \text { iv) } \beta_{i}=\bar{\gamma}_{i}, i=1,2, \quad \text { v) } e^{\theta_{1}+\eta_{1}}=e^{\bar{\theta}_{1}+\bar{\eta}_{1}}, \quad \text { vi) } e^{\theta_{1}+\eta_{2}}=e^{\bar{\theta}_{2}+\bar{\eta}_{1}}, \\
& \text { vii) } \left.e^{\theta_{2}+\eta_{1}}=e^{\bar{\theta}_{1}+\bar{\eta}_{2}}, \quad \text { viii) } e^{\theta_{2}+\eta_{2}}=e^{\bar{\theta}_{2}+\bar{\eta}_{2}}, \quad \text { ix }\right) e^{\alpha_{i j}}=e^{\bar{\alpha}_{j i}}, i, j=1,2, \\
& \text { x) } M=\bar{M}, \quad x i) e^{\theta_{1}+\theta_{2}+\eta_{1}+\eta_{2}}=e^{\bar{\theta}_{1}+\bar{\theta}_{2}+\bar{\eta}_{1}+\bar{\eta}_{2}} .
\end{aligned}
$$




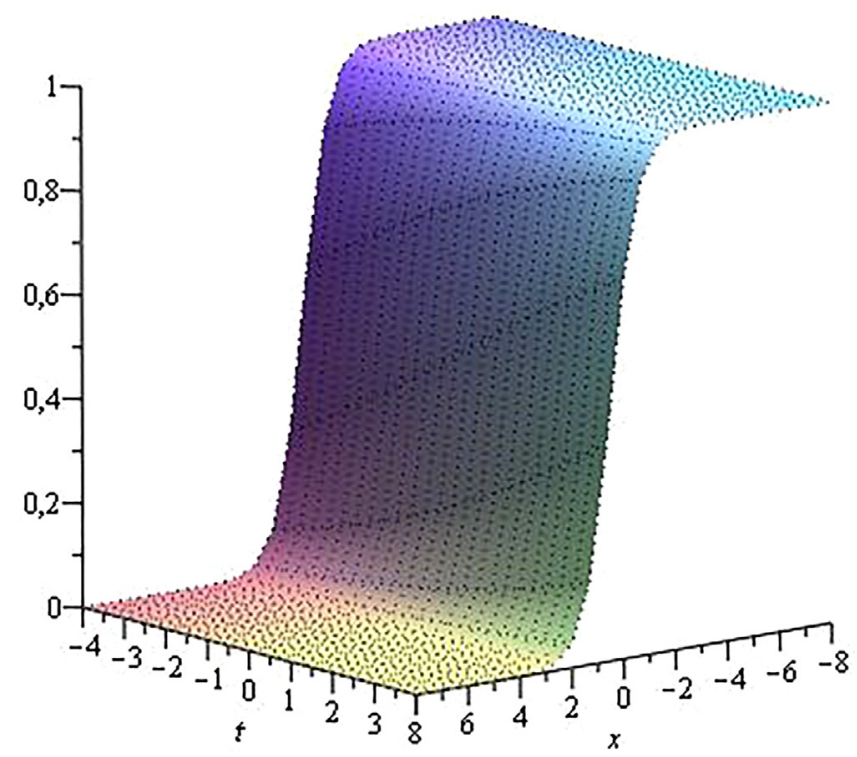

Fig. 5. Kink-type wave for (88) with the parameters $k_{1}=0, k_{2}=1, e^{\delta_{1}}=a_{1}+i b_{1}=i, e^{\delta_{2}}=a_{2}+i b_{2}=1, k=-1, a=2$.

From the condition $i$ ) we have $e^{\ell_{i} x-\frac{\ell_{i}^{3}}{4 a} t+\alpha_{i}}=k e^{\varepsilon_{2} \bar{k}_{i} x-\varepsilon_{1} \frac{\bar{k}_{i}^{3}}{4 a} t+\bar{\delta}_{i}}, i=1,2$ which gives $\ell_{i}=\varepsilon_{2} \bar{k}_{i}$ and $e^{\alpha_{i}}=k e^{\bar{\delta}_{i}}, i=1$, 2. Since

$$
-\frac{l_{i}^{3}}{4 a}=-\frac{\varepsilon_{2} \bar{k}_{i}^{3}}{4 \bar{a} \varepsilon_{1} \varepsilon_{2}}=-\frac{\bar{k}_{i}^{3}}{4 \bar{a}},
$$

the coefficients of $t$ are equal without any additional condition. The other conditions are also satisfied by the following constraints:

$$
\text { 1) } \left.\bar{a}=\varepsilon_{1} \varepsilon_{2} a, \quad \text { 2) } \ell_{i}=\varepsilon_{2} \bar{k}_{i}, \quad i=1,2, \quad 3\right) e^{\alpha_{i}}=k e^{\bar{\delta}_{i}}, \quad i=1,2 .
$$

Now by giving particular values to the parameters satisfying the constraints (95), we present some examples of twosoliton solutions for three different types of nonlocal cmKdV equations. Note that since the expressions for the real-valued function $|q(t, x)|^{2}$ are very long, we will only give the functions $q(t, x)$.

3.2.1. Case a. (T-symmetric): $r=k \bar{q}(-t, x)$

In this case we have $\bar{a}=-a, \ell_{i}=\bar{k}_{i}$, and $e^{\alpha_{i}}=k e^{\bar{\delta}_{i}}, i=1,2$. We give an example below for this case.

Example 9. For the parameters $\left(k_{1}, \ell_{1}, k_{2}, \ell_{2}\right)=\left(\frac{1}{4}, \frac{1}{4},-\frac{1}{2},-\frac{1}{2}\right)$ with $\left(e^{\alpha_{j}}, e^{\delta_{j}}, k, a\right)=(-1+i, 1+i,-1,10 i), j=1,2$, then the solution $|q(t, x)|^{2}$ becomes

$$
|q(t, x)|^{2}=\frac{A}{B},
$$

where

$$
\begin{aligned}
A= & e^{-\frac{1}{2} x}\left(288 \cos \left(\frac{1}{1280} t\right)+72 \cos \left(\frac{1}{160} t\right)\right)+\cos \left(\frac{7}{2560} t\right)\left(288 e^{\frac{1}{4} x}+72 e^{-\frac{5}{4} x}\right) \\
& +\cos \left(\frac{9}{2560} t\right)\left(5184 e^{-\frac{3}{4} x}+4 e^{-\frac{1}{4} x}\right)
\end{aligned}
$$

and

$$
\begin{aligned}
B= & 4096 e^{-\frac{1}{2} x}+4 e^{-2 x}+1679616 e^{-x}+64 e^{x}+1+\cos \left(\frac{1}{1280} t\right)\left(5184 e^{-\frac{3}{2} x}+16 e^{\frac{1}{2} x}\right) \\
& +\cos \left(\frac{1}{160} t\right)\left(20736+4 e^{-x}\right)+\cos \left(\frac{7}{2560} t\right)\left(165888 e^{-\frac{3}{4} x}+128 e^{-\frac{1}{4} x}\right) \\
& +\cos \left(\frac{9}{2560} t\right)\left(1024 e^{\frac{1}{4} x}+256 e^{-\frac{5}{4} x}\right)+e^{-\frac{1}{2} x}\left(2592 \cos \left(\frac{7}{1280} t\right)+32 \cos \left(\frac{9}{1280} t\right)\right) .
\end{aligned}
$$

The solution (96) is a singular function. 
3.2.2. Case $b$. (S-symmetric): $r=k \bar{q}(t,-x)$

This case gives $\bar{a}=-a, \ell_{i}=-\bar{k}_{i}$, and $e^{\alpha_{i}}=k e^{\bar{\delta}_{i}}, i=1,2$. Consider the following example.

Example 10. Choose the parameters as $\left(k_{1}, \ell_{1}, k_{2}, \ell_{2}\right)=\left(\frac{i}{4}, \frac{i}{4},-\frac{i}{2},-\frac{i}{2}\right)$ with $\left(e^{\alpha_{j}}, e^{\delta_{j}}, k, a\right)=(1,1,1,2 i), j=1,2$. Then the solution $|q(t, x)|^{2}$ becomes

$$
|q(t, x)|^{2}=\frac{A}{B}
$$

where

$$
\begin{aligned}
A= & e^{-\frac{3}{256} t}\left[81+e^{\frac{1}{80} t}+e^{\frac{7}{1280} t}+1296 e^{\frac{9}{1280} t}+\cos \left(\frac{1}{4} x\right)\left(72 e^{\frac{5}{512} t}+18 e^{\frac{7}{2560} t}\right)\right. \\
& \left.+\cos \left(\frac{3}{4} x\right)\left(2 e^{\frac{23}{2560} t}+648 e^{\frac{9}{2500} t}\right)+18 \cos x e^{\frac{1}{160} t}\right]
\end{aligned}
$$

and

$$
\begin{aligned}
B= & 1+1024 e^{-\frac{7}{1280} t}+104976 e^{-\frac{7}{640} t}+e^{-\frac{1}{80} t}+16 e^{\frac{1}{640} t}+\cos \left(\frac{1}{4} x\right)\left[64 e^{-\frac{7}{2560} t}+20736 e^{-\frac{21}{2560} t}\right] \\
& +\cos \left(\frac{1}{2} x\right)\left[648 e^{-\frac{7}{1280} t}+8 e^{\frac{1}{1280} t}+648 e^{-\frac{3}{256} t}\right]+8 e^{-\frac{7}{1280} t} \cos \left(\frac{3}{2} x\right) \\
& +\cos \left(\frac{3}{4} x\right)\left[256 e^{-\frac{1}{512} t}+64 e^{-\frac{23}{2560} t}\right]+\cos x\left[2 e^{-\frac{1}{160} t}+2592 e^{-\frac{3}{640} t}\right] .
\end{aligned}
$$

The solution (99) is singular.

3.2.3. Case c. (ST-symmetric): $r=k \bar{q}(-t,-x)$

In this case the parameters satisfy $\bar{a}=a, \ell_{i}=-\bar{k}_{i}$, and $e^{\alpha_{i}}=k e^{\bar{\delta}_{i}}, i=1,2$. Here we give the following example.

Example 11. For the parameters $\left(k_{1}, \ell_{1}, k_{2}, \ell_{2}\right)=(2 i, 2 i, i, i)$ with $\left(e^{\alpha_{j}}, e^{\delta_{j}}, k, a\right)=(1,1,1,1), j=1,2$, we have the solution $|q(t, x)|^{2}$

$$
|q(t, x)|^{2}=\frac{A}{B}
$$

where

$$
A=\frac{5185}{2592} \cos \left(x+\frac{7}{4} t\right)+\frac{5}{72} \cos \left(3 x+\frac{9}{4} t\right)+\frac{1}{18} \cos \left(2 x+\frac{1}{2} t\right)+\frac{1}{72} \cos (4 x+4 t)+\frac{41489}{20736},
$$

and

$$
\begin{aligned}
B= & \frac{5}{36} \cos \left(x+\frac{7}{4} t\right)+\frac{1}{32} \cos \left(2 x+\frac{7}{2} t\right)+\frac{5185}{11664} \cos \left(3 x+\frac{9}{4} t\right)+\frac{1}{2592} \cos \left(6 x+\frac{9}{2} t\right) \\
& +\frac{20737}{41472} \cos \left(2 x+\frac{1}{2} t\right)+\frac{1297}{10368} \cos (4 x+4 t)+\frac{29985553}{26873856}
\end{aligned}
$$

The graph of the solution (100) is given in Fig. 6. It represents a periodical breather-type wave solution.

3.3. Three-soliton solution for the nonlocal CMKdV equations:

$\left(r=k \bar{q}\left(\varepsilon_{1} t, \varepsilon_{2} x\right)\right)$

We first find the conditions on the parameters of three-soliton solution of the mKdV system to satisfy the equality (7) where $r(t, x)$ is given by (50) and

$$
k \bar{q}\left(\varepsilon_{1} t, \varepsilon_{2} x\right)=k \frac{e^{\bar{\theta}_{1}}+e^{\bar{\theta}_{2}}+e^{\bar{\theta}_{3}}+\sum_{\substack{1 \leq i, j, s \leq 3 \\ i<j}} \bar{A}_{i j s} e^{\bar{\theta}_{i}+\bar{\theta}_{j}+\bar{\eta}_{s}}+\sum_{\substack{1 \leq i, j \leq 3 \\ i<j}} \bar{V}_{i j} e^{\bar{\theta}_{1}+\bar{\theta}_{2}+\bar{\theta}_{3}+\bar{\eta}_{i}+\bar{\eta}_{j}}}{1+\sum_{1 \leq i, j \leq 3} e^{\bar{\theta}_{i}+\bar{\eta}_{j}+\bar{\alpha}_{i j}}+\sum_{\substack{1 \leq i<j \leq 3 \\ 1 \leq p<r \leq 3}} \bar{M}_{i j p r} e^{\bar{\theta}_{i}+\bar{\theta}_{j}+\bar{\eta}_{p}+\bar{\eta}_{r}}+\bar{H} e^{\bar{\theta}_{1}+\bar{\theta}_{2}+\bar{\theta}_{3}+\bar{\eta}_{1}+\bar{\eta}_{2}+\bar{\eta}_{3}},},
$$

where

$$
\begin{aligned}
& \bar{\theta}_{i}=\varepsilon_{2} \bar{k}_{i} x-\varepsilon_{1} \frac{\bar{k}_{i}^{3}}{4 \bar{a}} t+\bar{\delta}_{i}, i=1,2,3, \\
& \bar{\eta}_{i}=\varepsilon_{2} \bar{\ell}_{i} x-\varepsilon_{1} \frac{\bar{\ell}_{i}^{3}}{4 \bar{a}} t+\bar{\alpha}_{i}, i=1,2,3 .
\end{aligned}
$$

Here we obtain that (7) is satisfied by the following conditions:

1) $\left.\left.\bar{a}=\varepsilon_{1} \varepsilon_{2} a, \quad 2\right) \ell_{i}=\varepsilon_{2} \bar{k}_{i}, \quad i=1,2,3, \quad 3\right) e^{\alpha_{i}}=k e^{\bar{\delta}_{i}}, \quad i=1,2,3$.

Now we will present some examples of three-soliton solutions of the nonlocal reductions of the mKdV system for particular parameters. 


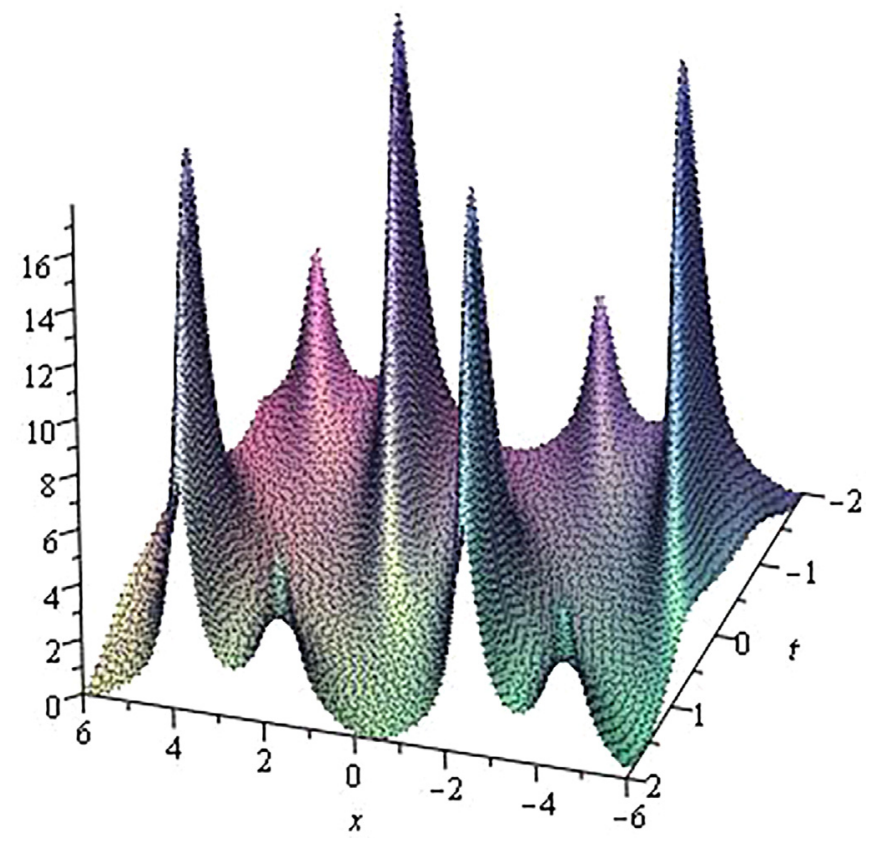

Fig. 6. A periodical breather-type wave plotted for (100) with the parameters $k_{1}=\ell_{1}=2 i, k_{2}=\ell_{2}=i, e^{\alpha_{j}}=e^{\delta_{j}}=k=a=1, j=1,2$.

\subsubsection{Case a. (T-Symmetric): $r=k \bar{q}(-t, x)$}

Here the parameters satisfy $\bar{a}=-a, \ell_{i}=\bar{k}_{i}$, and $e^{\alpha_{i}}=k e^{\bar{\delta}_{i}}, i=1,2,3$. We give the following example.

Example 12. For the set of parameters given by $\left(k_{1}, l_{1}, k_{2}, l_{2}, k_{3}, l_{3}\right)=\left(\frac{1}{4}, \frac{1}{4},-\frac{1}{2},-\frac{1}{2},-\frac{1}{6},-\frac{1}{6}\right)$ with $\left(e^{\alpha_{j}}, e^{\delta_{j}}, k, a\right)=(-1+$ $i, 1+i,-1,10 i), j=1,2,3$ the solution $q(t, x)$ becomes

$$
q(t, x)=\frac{A}{B},
$$

where

$$
\begin{aligned}
A= & (1+i)\left[e^{\frac{1}{4} x+\frac{1}{2500} i t}+e^{-\frac{1}{2} x-\frac{1}{320} i t}+e^{-\frac{1}{6} x-\frac{1}{8640} i t}+72 e^{-\frac{3}{1280} i t}+18 e^{-\frac{3}{4} x-\frac{3}{512} i t}\right. \\
& +889 e^{-\frac{5}{12} x-\frac{197}{69120} i t}+200 e^{\frac{1}{3} x+\frac{23}{34560} i t}+450 e^{-\frac{1}{12} x+\frac{11}{69120} i t}+\frac{1}{2} e^{-\frac{7}{6} x-\frac{11}{1728} i t} \\
& \left.+\frac{9}{2} e^{-\frac{5}{6} x-\frac{29}{8640} i t}+5400 e^{-\frac{2}{3} x-\frac{193}{34560} i t}+202500 e^{-\frac{1}{3} x-\frac{89}{34560} i t}+\frac{2025}{4} e^{-\frac{13}{12} x-\frac{421}{6912} i t}\right]
\end{aligned}
$$

and

$$
\begin{aligned}
B= & 1+9 e^{-\frac{2}{3} x-\frac{7}{2160} i t}+64 e^{-\frac{1}{4} x-\frac{7}{2560} i t}+2592 e^{-\frac{11}{12} x-\frac{413}{69120} i t}+1296 e^{-\frac{1}{2} x-\frac{7}{1280} i t} \\
& +2 e^{-x-\frac{1}{160} i t}+16200 e^{-\frac{1}{6} x-\frac{17}{6912} i t}+18 e^{-\frac{1}{3} x-\frac{1}{4320} i t}+8 e^{\frac{1}{2} x+\frac{1}{1280} i t}+\frac{9}{4} e^{-\frac{4}{3} x-\frac{7}{1080} i t} \\
& +903474 e^{-\frac{5}{6} x-\frac{197}{34500} i t}+7200 e^{-\frac{7}{12} x-\frac{41}{13824} i t}+576 e^{\frac{1}{12} x+\frac{19}{69120} i t}+90000 e^{\frac{1}{6} x+\frac{19}{34560} i t},
\end{aligned}
$$

which is a singular solution.

3.3.2. Case b. (S-symmetric): $r=k \bar{q}(t,-x)$

In this case we have $\bar{a}=-a, \ell_{i}=-\bar{k}_{i}$, and $e^{\alpha_{i}}=k e^{\bar{\delta}_{i}}, i=1,2,3$. Consider the following example.

Example 13. Let us take the set of parameters as: $\left(k_{1}, l_{1}, k_{2}, l_{2}, k_{3}, l_{3}\right)=\left(\frac{i}{4}, \frac{i}{4},-\frac{i}{2},-\frac{i}{2},-\frac{i}{6},-\frac{i}{6}\right)$ with $\left(e^{\alpha_{j}}, e^{\delta_{j}}, k, a\right)=$ $\left(1,1,1, \frac{i}{2}\right), j=1,2,3$. Then we have the solution $q(t, x)$

$$
q(t, x)=\frac{A}{B},
$$

where

$$
A=e^{\frac{1}{4} i x+\frac{1}{128} t}+e^{-\frac{1}{2} i x-\frac{1}{16} t}+e^{-\frac{1}{6} i x-\frac{1}{432} t}+36 e^{-\frac{3}{64} t}+9 e^{-\frac{3}{4} i x-\frac{15}{128} t}
$$




$$
\begin{aligned}
& +\frac{889}{2} e^{-\frac{5}{12} i x-\frac{197}{3456} t}+100 e^{\frac{1}{3} i x+\frac{23}{1728} t}+225 e^{-\frac{1}{12} i x+\frac{11}{3456} t}+\frac{1}{4} e^{-\frac{7}{6} i x-\frac{55}{432} t} \\
& +\frac{9}{4} e^{-\frac{5}{6} i x-\frac{29}{432} t}+1350 e^{-\frac{2}{3} i x-\frac{193}{1728} t}+50625 e^{-\frac{1}{3} i x-\frac{89}{1728} t}+\frac{2025}{16} e^{-\frac{13}{12} i x-\frac{421}{3456} t}
\end{aligned}
$$

and

$$
\begin{aligned}
B= & 1+4 e^{\frac{1}{2} i x+\frac{1}{64} t}+32 e^{-\frac{1}{4} i x-\frac{7}{128} t}+288 e^{\frac{1}{12} i x+\frac{19}{3456} t}+e^{-i x-\frac{1}{8} t}+\frac{9}{2} e^{-\frac{2}{3} i x-\frac{7}{108} t} \\
& +9 e^{-\frac{1}{3} i x-\frac{1}{216} t}+648 e^{-\frac{11}{12} i x-\frac{413}{3456} t}+4050 e^{-\frac{1}{6} i x-\frac{85}{1728} t}+1800 e^{-\frac{7}{12} i x-\frac{205}{3456} t} \\
& +324 e^{-\frac{1}{2} i x-\frac{7}{64} t}+22500 e^{\frac{1}{6} i x+\frac{19}{1728} t}+\frac{9}{16} e^{-\frac{4}{3} i x-\frac{7}{54} t}+\frac{451737}{4} e^{-\frac{5}{6} i x-\frac{197}{1728} t} .
\end{aligned}
$$

It is a singular solution.

\subsubsection{Case c. (ST-symmetric): $r=k \bar{q}(-t,-x)$}

Here we have $\bar{a}=a, \ell_{i}=-\bar{k}_{i}$, and $e^{\alpha_{i}}=k e^{\bar{\delta}_{i}}, i=1,2,3$. We give the following example.

Example 14. Consider the following set of the parameters: $\left(k_{1}, l_{1}, k_{2}, l_{2}, k_{3}, l_{3}\right)=\left(\frac{i}{4}, \frac{i}{4}, \frac{i}{2}, \frac{i}{2},-\frac{i}{6},-\frac{i}{6}\right)$ with $\left(e^{\alpha_{j}}, e^{\delta_{j}}, k, a\right)=$ $(-1+i, 1+i,-1,2), j=1,2,3$. Then the solution $q(t, x)$ becomes

$$
q(t, x)=\frac{A}{B}
$$

where

$$
\begin{aligned}
A= & (1+i)\left[e^{\frac{1}{4} i x+\frac{1}{512} i t}+e^{\frac{1}{2} i x+\frac{1}{64} i t}+e^{-\frac{1}{6} i x-\frac{1}{1728} i t}-\frac{8}{9} e^{i x+\frac{5}{256} i t}-\frac{2}{9} e^{\frac{5}{4} i x+\frac{17}{512} i t}\right. \\
& -\frac{3556}{9} e^{\frac{7}{12} i x+\frac{235}{13824} i t}-200 e^{\frac{1}{3} i x+\frac{23}{6912} i t}-450 e^{-\frac{1}{12} i x+\frac{11}{13824} i t}-8 e^{\frac{5}{6} i x+\frac{53}{1728} i t} \\
& \left.-72 e^{\frac{1}{6} i x+\frac{25}{1728} i t}+\frac{3200}{243} e^{\frac{4}{3} i x+\frac{239}{6912} i t}+40000 e^{\frac{2}{3} i x+\frac{127}{6912} i t}+1600 e^{\frac{11}{12} i x+\frac{443}{13824} i t}\right]
\end{aligned}
$$

and

$$
\begin{aligned}
B= & -18 e^{-\frac{1}{3} i x-\frac{1}{864} i t}+90000 e^{\frac{1}{6} i x+\frac{19}{6912} i t}+512 e^{\frac{13}{12} i x+\frac{451}{13824} i t}-\frac{64}{9} e^{\frac{3}{4} i x+\frac{9}{512} i t} \\
& +576 e^{\frac{2}{3} i x+\frac{13}{432} i t}-36 e^{\frac{1}{3} i x+\frac{13}{864} i t}-576 e^{\frac{1}{12} i x+\frac{19}{13824} i t}-2 e^{i x+\frac{1}{32} i t}+800 e^{\frac{5}{6} i x+\frac{131}{6912} i t} \\
& +12800 e^{\frac{5}{12} i x+\frac{227}{13824} i t}-8 e^{\frac{1}{2} i x+\frac{1}{256} i t}-\frac{47027200}{1323} e^{\frac{7}{6} i x+\frac{235}{6912} i t}+\frac{16}{81} e^{\frac{3}{2} i x+\frac{9}{256} i t} .
\end{aligned}
$$

The graph of the solution (107) is given in Fig. 7.

3.4. One-soliton solution for the nonlocal MKdV equations: $r=k q(-t,-x)$

When we apply the nonlocal reduction $r(t, x)=k q\left(\varepsilon_{1} t, \varepsilon_{2} x\right)$ to the mKdV system (1) and (2) we obtain the equation

$$
a q_{t}(t, x)=-\frac{1}{4} q_{x x x}(t, x)+\frac{3}{2} k q(t, x) q\left(\varepsilon_{1} t, \varepsilon_{2} x\right) q_{x}(t, x)
$$

provided that $\varepsilon_{1} \varepsilon_{2}=1$ which is possible when $\left(\varepsilon_{1}, \varepsilon_{2}\right)=(1,1)$ or $\left(\varepsilon_{1}, \varepsilon_{2}\right)=(-1,-1)$. The first case gives a local equation. By the latter one the nonlocal reduction becomes $r=k q(-t,-x)$ and we get the nonlocal ST-symmetric mKdV equation (14).

If we consider one-soliton solution (24) obtained by the Hirota direct method with the reduction $r=k q(-t,-x)$ through the Type 1 approach, we get $k_{1}=-k_{2}$ which gives trivial solution $q(t, x)=0$. Thus we will use the Type 2 approach to find nontrivial one-soliton solution of the nonlocal equation (14).

We have

$$
\frac{e^{\theta_{2}}}{1+A e^{\theta_{1}+\theta_{2}}}=k \frac{e^{\theta_{1}^{-}}}{1+A e^{\theta_{1}^{-}+\theta_{2}^{-}}},
$$

so from the application of the cross multiplication we get

$$
e^{\theta_{2}}+A e^{2 \delta_{2}} e^{\theta_{1}^{-}}=k e^{\theta_{1}^{-}}+A k e^{2 \delta_{1}} e^{\theta_{2}},
$$

where

$$
\theta_{j}=k_{j} x-\frac{k_{j}^{3}}{4 a} t+\delta_{j}, \quad \theta_{1}^{-}=-k_{j} x+\frac{k_{j}^{3}}{4 a} t+\delta_{j}, \quad j=1,2 .
$$

Hence we obtain the conditions

$$
\text { i) } A k e^{2 \delta_{1}}=1, \quad \text { ii) } A e^{2 \delta_{2}}=k \text {, }
$$




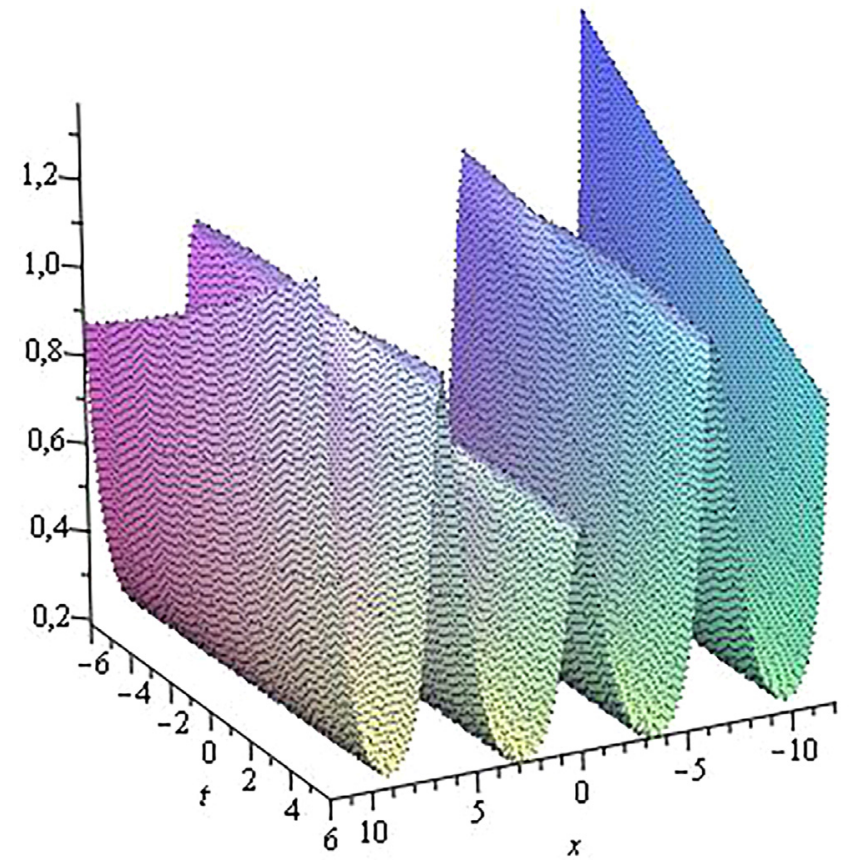

Fig. 7. A periodical wave plotted for $|q(t, x)|^{2}$ corresponding to (107) with the parameters $k_{1}=\ell_{1}=\frac{i}{4}, k_{2}=\ell_{2}=\frac{i}{2}, k_{3}=\ell_{3}=-\frac{i}{6}, e^{\alpha_{j}}=-1+i, e^{\delta_{j}}=1+i$, $j=1,2,3, k=-1, a=2$.

yielding $e^{\delta_{1}}= \pm i \frac{\left(k_{1}+k_{2}\right)}{\sqrt{k}}$ and $e^{\delta_{2}}= \pm i \sqrt{k}\left(k_{1}+k_{2}\right)$. Therefore one-soliton solution of the Eq. (14) is

$$
q(t, x)=\frac{i \sigma_{1} e^{k_{1} x-\frac{k_{1}^{3} t}{4 a} t}\left(k_{1}+k_{2}\right)}{\sqrt{k}\left(1+\sigma_{1} \sigma_{2} e^{\left(k_{1}+k_{2}\right) x-\frac{\left(k_{1}^{3}+k_{2}^{3}\right)}{4 a} t}\right)}, \quad \sigma_{j}= \pm 1, j=1,2 .
$$

Let $a \in \mathbb{R}, k_{1}=\alpha_{1}+i \beta_{1}$, and $k_{2}=\alpha_{2}+i \beta_{2}$. Then the solution $|q(t, x)|^{2}$ corresponding to (112) becomes

$$
|q(t, x)|^{2}=\frac{e^{\theta}\left(\left(\alpha_{1}+\alpha_{2}\right)^{2}+\left(\beta_{1}+\beta_{2}\right)^{2}\right)}{2 k\left[\cosh (\phi)+\sigma_{1} \sigma_{2} \cos (\varphi)\right]},
$$

where $\theta=\left(\alpha_{1}-\alpha_{2}\right) x-\frac{\alpha_{1}^{3}-3 \alpha_{1} \beta_{1}^{2}-\alpha_{2}^{3}+3 \alpha_{2} \beta_{2}^{2}}{4 a} t, \phi=A_{1} x+B_{1} t$, and $\varphi=A_{2} x+B_{2} t$. Here

$$
\begin{aligned}
& A_{1}=\alpha_{1}+\alpha_{2}, \quad B_{1}=-\frac{\left(\alpha_{1}^{3}-3 \alpha_{1} \beta_{1}^{2}+\alpha_{2}^{3}-3 \alpha_{2} \beta_{2}^{2}\right)}{4 a}, \\
& A_{2}=\beta_{1}+\beta_{2}, \quad B_{2}=\frac{\left(\beta_{1}^{3}-3 \alpha_{1}^{2} \beta_{1}+\beta_{2}^{3}-3 \alpha_{2}^{2} \beta_{2}\right)}{4 a} .
\end{aligned}
$$

There are cases where the solution (113) is nonsingular.

(a) If we have $k_{1}=k_{2}$ for real $k_{1}$ and $\sigma_{1} \sigma_{2}=1$ then the solution (112) becomes

$$
q(t, x)=\frac{i \sigma_{1} k_{1}}{\sqrt{k}} \operatorname{sech}\left(k_{1} x-\frac{k_{1}^{3}}{4 a} t\right) .
$$

Assuming $a=\frac{1}{4}, k=-1, k_{1}=-2 \eta$, and $k_{2}=-2 \bar{\eta}$, the solution (112) is reduced to the one given by Ablowitz and Musslimani [8]. In [26], Ji and Zhu also considered the Eq. (14) and found one- and two-soliton solution of this equation by using the Darboux transformation. The solution (112) can be transformed to the one-soliton solution in [26] by taking $a=\frac{1}{4}$, $k=-1, k_{1}=4 i \mu_{1}$, and $k_{2}=-2 v_{1}+2 i \mu_{1}$. Besides this type of solution they obtained rogue-wave and rational solutions of (14). In [27], Ji and Zhu obtained the same one-soliton solution through inverse scattering transform. Let us now consider some examples.

Example 15. Consider the following set of the parameters $\left(k_{1}, k_{2}, \sigma_{1}, \sigma_{2}, k, a\right)=\left(\frac{1}{2}, \frac{1}{2}, 1,1,-1,2\right)$. Then the solution becomes

$$
q(t, x)=\frac{e^{\frac{1}{2} x-\frac{1}{64} t}}{1+e^{x-\frac{1}{32} t}}=\frac{1}{2} \operatorname{sech}\left(\frac{1}{2} x-\frac{1}{64} t\right) .
$$




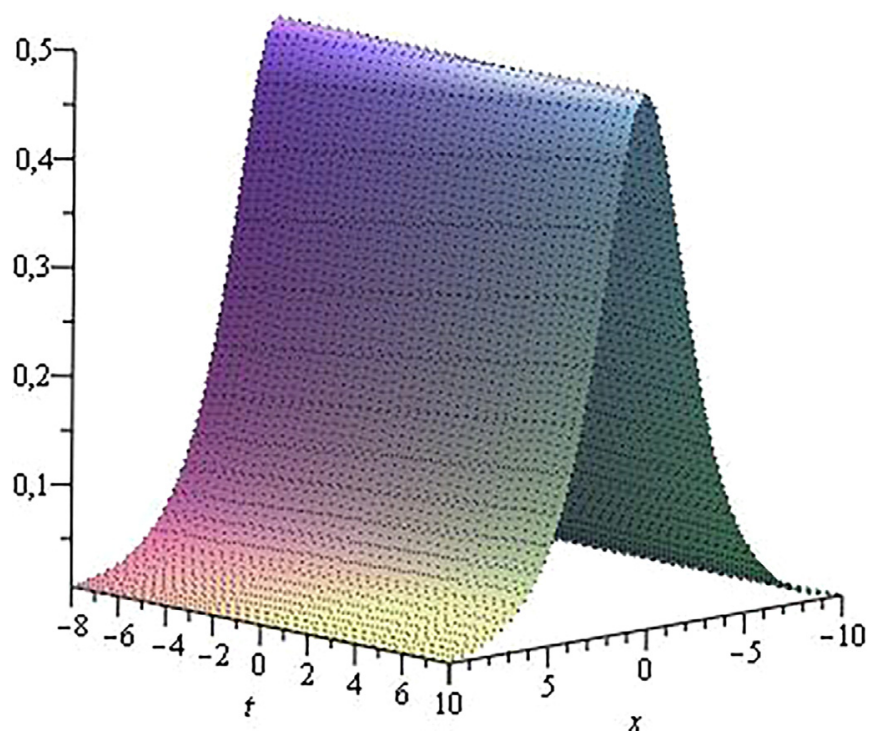

Fig. 8. One-soliton for (112) with the parameters $k_{1}=k_{2}=\frac{1}{2}, \sigma_{1}=\sigma_{2}=1, k=-1, a=2$.

It is one-soliton solution. The graph of (115) is given in Fig. 8.

(b) If $B_{1} A_{2}=B_{2} A_{1}$ then the solution (113) becomes

$$
|q(t, x)|^{2}=\frac{e^{\theta}\left(\left(\alpha_{1}+\alpha_{2}\right)^{2}+\left(\beta_{1}+\beta_{2}\right)^{2}\right)}{2 k\left[\cosh (\phi)+\sigma_{1} \sigma_{2} \cos \left(\frac{B_{2}}{B_{1}} \phi\right)\right]} .
$$

Example 16. Take $\left(k_{1}, k_{2}, \sigma_{1}, \sigma_{2}, k, a\right)=\left(i, 1+\frac{i}{2}, 1,1,-1, \frac{1}{4}\right)$. Then we have the one-soliton solution

$$
q(t, x)=\frac{\left(1+\frac{3}{2} i\right) e^{i x+i t}}{1+e^{\left(1+\frac{3}{2} i\right) x-\left(\frac{1}{4}+\frac{3}{8} i\right) t}}
$$

so

$$
|q(t, x)|^{2}=\frac{13 e^{-u}}{8\left[\cosh (u)+\cos \left(\frac{3}{2} u\right)\right]},
$$

where $u=x-\frac{1}{4} t$. This is a complexiton solution. The graph of (118) is given in Fig. 9.

3.5. Two-soliton solution for the nonlocal MKdV equations: $r=k q(-t,-x)$

Similar to the one-soliton solution case, if we use two-soliton solution (34)-(35) with the nonlocal reduction $r=$ $k q(-t,-x)$ and apply cross multiplication we obtain the following constraints to be satisfied by two-soliton solution of (14),

$$
e^{\delta_{j}}=\tau_{r} i \frac{\left(k_{j}+\ell_{1}\right)\left(k_{j}+\ell_{2}\right)}{\sqrt{k}\left(k_{1}-k_{2}\right)}, \quad e^{\alpha_{j}}=\rho_{r} i \sqrt{k} \frac{\left(k_{1}+\ell_{j}\right)\left(k_{2}+\ell_{j}\right)}{\left(\ell_{1}-\ell_{2}\right)}, \tau_{r}= \pm 1, \rho_{r}= \pm 1, r=1,2,
$$

for $j=1,2$. Hence two-soliton solution of (14) is

$$
q(t, x)=\frac{A}{B},
$$

where

$$
\begin{aligned}
A= & \frac{i}{\sqrt{k}\left(k_{1}-k_{2}\right)}\left[\tau_{1}\left(k_{1}+\ell_{2}\right)\left(k_{1}+\ell_{1}\right) e^{k_{1} x-\frac{k_{1}^{3}}{4 a} t}+\tau_{2}\left(k_{2}+\ell_{2}\right)\left(k_{2}+\ell_{1}\right) e^{k_{2} x-\frac{k_{2}^{3} t}{4 a} t}\right] \\
& +\frac{i \tau_{1} \tau_{2}}{\sqrt{k}\left(\ell_{1}-\ell_{2}\right)}\left[\left(k_{1}+\ell_{2}\right)\left(k_{2}+\ell_{2}\right) \rho_{1} e^{\left(k_{1}+k_{2}+\ell_{1}\right) x-\frac{\left(k_{1}^{3}+k_{2}^{3}+\ell_{1}^{3}\right)}{4 a} t}\right. \\
& \left.+\left(k_{1}+\ell_{1}\right)\left(k_{2}+\ell_{1}\right) \rho_{2} e^{\left(k_{1}+k_{2}+\ell_{2}\right) x-\frac{\left(k_{1}^{3}+k_{2}^{3}+\ell_{2}^{3}\right)}{4 a} t}\right]
\end{aligned}
$$

and

$$
B=1+\frac{1}{\left(k_{1}-k_{2}\right)\left(\ell_{1}-\ell_{2}\right)}\left[\tau_{1} \rho_{1}\left(k_{1}+\ell_{2}\right)\left(k_{2}+\ell_{1}\right) e^{\left(k_{1}+\ell_{1}\right) x-\frac{\left(k_{1}^{3}+\ell_{1}^{3}\right)}{4 a} t}\right.
$$




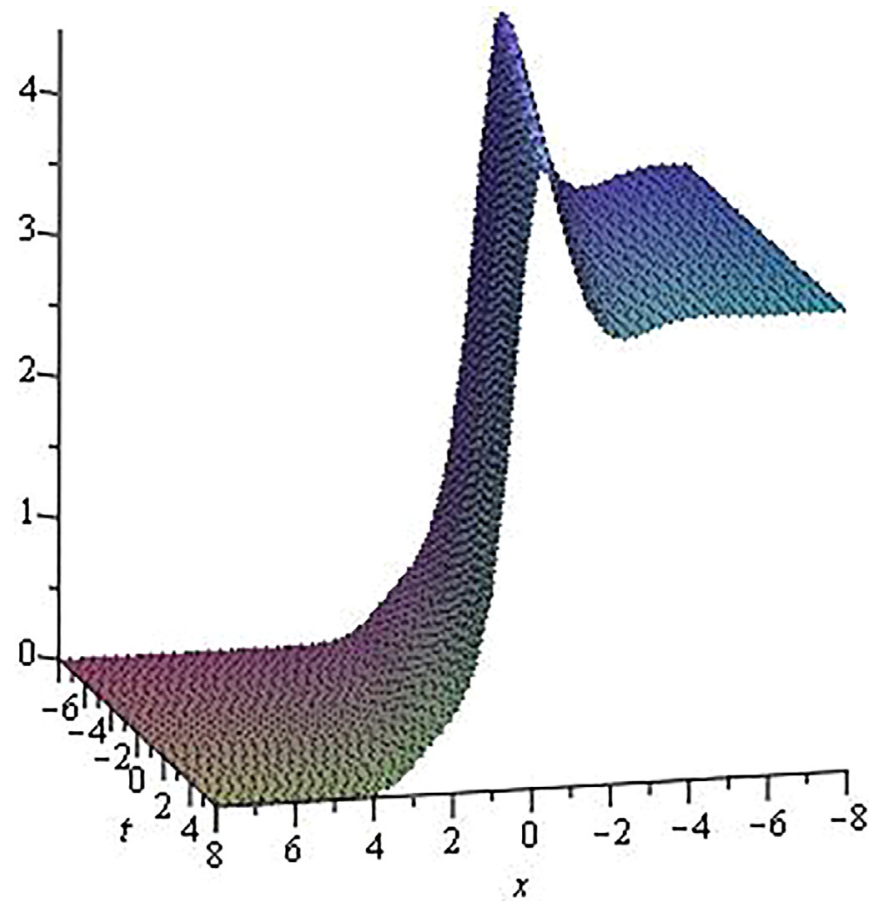

Fig. 9. A complexiton for $|q(t, x)|^{2}$ corresponding to (112) with the parameters $k_{1}=i, k_{2}=1+\frac{i}{2}, \sigma_{1}=\sigma_{2}=1, k=-1, a=\frac{1}{4}$.

$$
\begin{aligned}
& +\tau_{1} \rho_{2}\left(k_{1}+\ell_{1}\right)\left(k_{2}+\ell_{2}\right) e^{\left(k_{1}+\ell_{2}\right) x-\frac{\left(k_{1}^{3}+\ell_{2}^{3}\right)}{4 a} t}+\tau_{2} \rho_{1}\left(k_{2}+\ell_{2}\right)\left(k_{1}+\ell_{1}\right) e^{\left(k_{2}+\ell_{1}\right) x-\frac{\left(k_{2}^{3}+\ell_{1}^{3}\right)}{4 a} t} \\
& \left.+\tau_{2} \rho_{2}\left(k_{2}+\ell_{1}\right)\left(k_{1}+\ell_{2}\right) e^{\left(k_{2}+\ell_{2}\right) x-\frac{\left(k_{2}^{3}+\ell_{2}^{3}\right)}{4 a} t}\right]+\tau_{1} \tau_{2} \rho_{1} \rho_{2} e^{\left(k_{1}+k_{2}+\ell_{1}+\ell_{2}\right) x-\frac{\left(k_{1}^{3}+k_{2}^{3}+\ell_{1}^{3}+\ell_{2}^{3}\right)}{4 a} t} .
\end{aligned}
$$

Now we consider some particular examples.

Example 17. Take the parameters as $\left(k_{1}, k_{2}, \ell_{1}, \ell_{2}, k, a\right)=\left(1, \frac{1}{2}, 1, \frac{1}{2},-1, \frac{1}{4}\right)$ with $\tau_{j}=\rho_{j}=1, j=1,2$. The solution becomes

$$
q(t, x)=\frac{6 e^{x-t}+3 e^{\frac{1}{2} x-\frac{1}{8} t}+3 e^{\frac{5}{2} x-\frac{17}{8} t}+6 e^{2 x-\frac{5}{4} t}}{1+9 e^{2 x-2 t}+16 e^{\frac{3}{2} x-\frac{9}{8} t}+9 e^{x-\frac{1}{4} t}+e^{3 x-\frac{9}{4} t}} .
$$

This is a non-singular solution and its graph is given in Fig. 10.

Example 18. Consider the following set of parameters: $\left(k_{1}, k_{2}, \ell_{1}, \ell_{2}, k, a\right)=\left(1,0,1, \frac{1}{2},-1, \frac{1}{4}\right)$ with $\tau_{j}=\rho_{j}=1, j=1,2$. We have the solution

$$
q(t, x)=\frac{1+3 e^{x-t}+\frac{3}{2} e^{2 x-2 t}+4 e^{\frac{3}{2} x-\frac{9}{8} t}}{1+3 e^{2 x-2 t}+2 e^{\frac{3}{2} x-\frac{9}{8} t}+2 e^{x-t}+3 e^{\frac{1}{2} x-\frac{1}{8} t}+e^{\frac{5}{2} x-\frac{17}{8} t}} .
$$

The graph of (124) is given in Fig. 11. It represents interaction of soliton and kink-type wave.

Example 19. Choose $\left(k_{1}, k_{2}, \ell_{1}, \ell_{2}, k, a\right)=\left(1,0,2, \frac{1}{2},-1, \frac{1}{4}\right)$ with $\tau_{j}=\rho_{j}=-1, j=1,2$. The two-soliton solution becomes

$$
q(t, x)=-\frac{1+\frac{9}{2} e^{x-t}+\frac{1}{2} e^{3 x-9 t}+4 e^{\frac{3}{2} x-\frac{9}{8} t}}{1+2 e^{3 x-9 t}+e^{\frac{3}{2} x-\frac{9}{8} t}+e^{2 x-8 t}+2 e^{\frac{1}{2} x-\frac{1}{8} t}+e^{\frac{7}{2} x-\frac{73}{8} t}}
$$

The graph of the above solution is given in Fig. 12. This solution represents interaction of soliton and kink-type wave.

Example 20. Choose $\left(k_{1}, k_{2}, \ell_{1}, \ell_{2}, k, a\right)=\left(1+\frac{i}{2},-1+\frac{i}{2}, 1+\frac{i}{2},-1+\frac{i}{2},-1, \frac{1}{4}\right)$ with $\tau_{j}=\rho_{j}=1, j=1,2$. Then the twosoliton solution becomes

$$
q(t, x)=\frac{8 \sinh \left(x-\frac{1}{4} t\right) \sin \left(\frac{x}{2}-\frac{11}{8} t\right)-4 \cosh \left(x-\frac{1}{4} t\right) \cos \left(\frac{x}{2}-\frac{11}{8} t\right)}{4 \cos \left(x-\frac{11}{4} t\right)-\cosh \left(2 x-\frac{t}{2}\right)-5}
$$

This solution is a breather-type wave solution. The graph of (126) is given in Fig. 13.

Note that in [26,27], Ji and Zhu also obtained two-soliton solution of the Eq. (14) representing interactions of brightbright solitons, bright-dark solitons, soliton-kink, and also breather solutions, by using the Darboux transformation and 


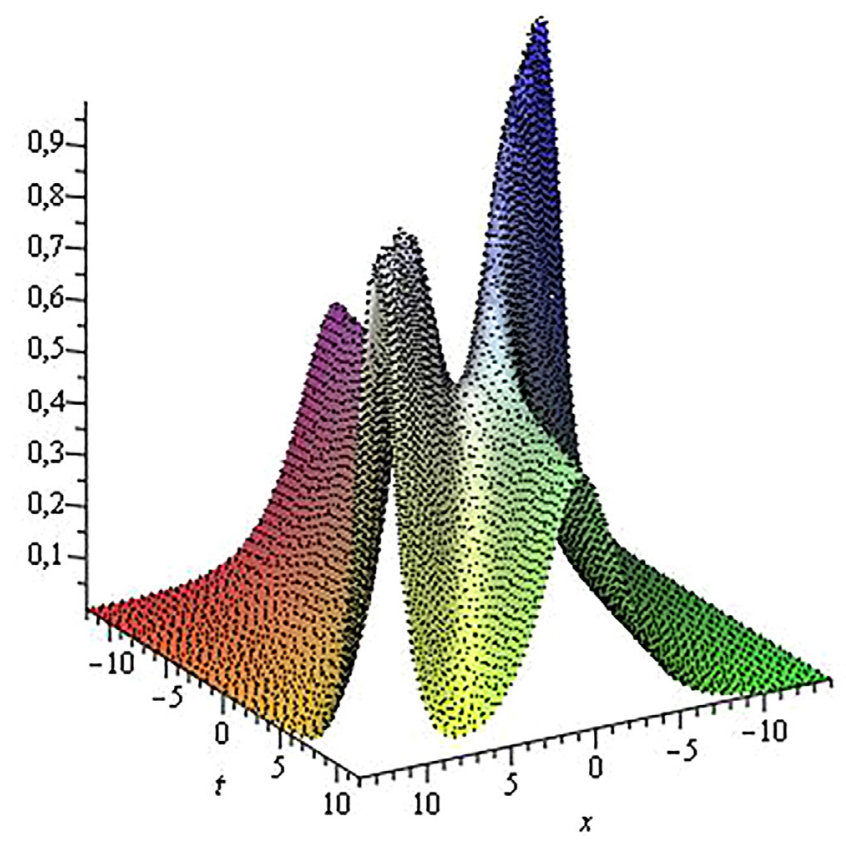

Fig. 10. Two-solitons for (120) with the parameters $k_{1}=\ell_{1}=1, k_{2}=\ell_{2}=\frac{1}{2}, k=-1, a=\frac{1}{4}, \tau_{j}=\rho_{j}=1, j=1,2$.

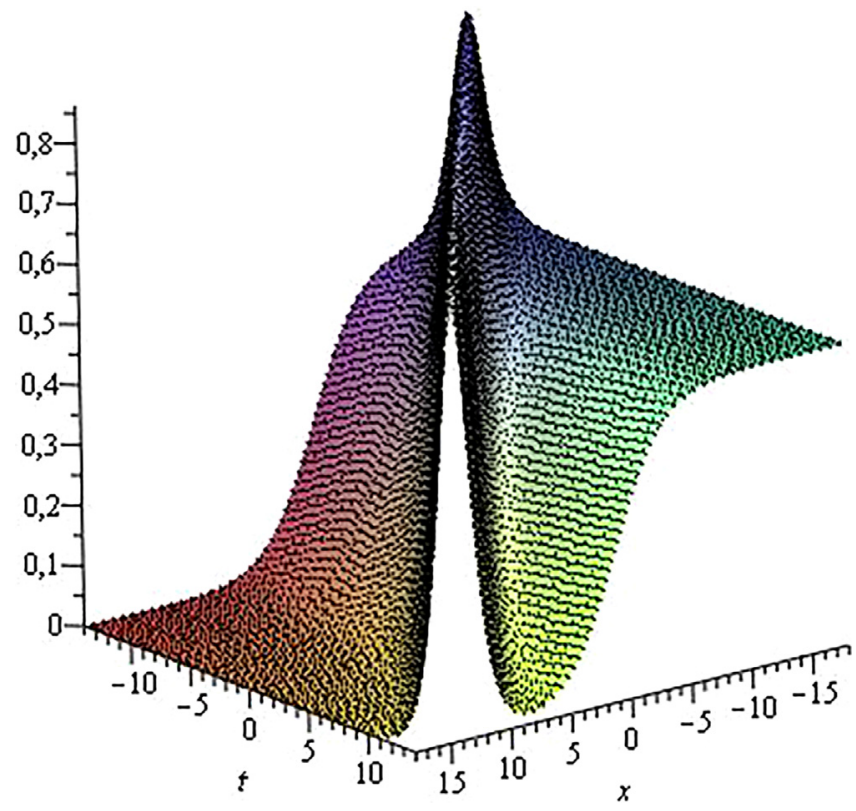

Fig. 11. Soliton and kink-type wave for (120) with the parameters $k_{1}=\ell_{1}=1, k_{2}=0, \ell_{2}=\frac{1}{2}, k=-1, a=\frac{1}{4}, \tau_{j}=\rho_{j}=1, j=1,2$.

inverse scattering transform, respectively. Actually, if we let $a=\frac{1}{4}, k=-1, k_{1}=-2 v_{2}-2 i \mu_{2}, k_{2}=2 v_{2}-2 i \mu_{2}, l_{1}=-2 v_{1}+$ $2 i \mu_{1}, l_{2}=2 v_{1}+2 i \mu_{1}$, and $\rho_{j}=\tau_{j}=1, j=1,2$, our solution (120) with (121) and (122) can be transformed to the twosoliton solution given in Ref. [26]. Similarly, assuming $a=\frac{1}{4}, k=-1$, and using the notations for the parameters in Ref. [27] as $k_{1}=-2 i \bar{k}_{1}, k_{2}=-2 i \bar{k}_{2}, l_{1}=2 i k_{1}, l_{2}=2 i k_{2}$, and $\tau_{j}=\bar{\sigma}_{j}, \rho_{j}=\sigma_{j}, j=1,2$, our two-soliton solution turns to be the solution given in Ref. [27].

\section{Concluding remarks}

In this work, we have studied nonlocal reductions of the mKdV system of nonlinear equations. There are two nonlocal reductions of these equations. Nonlocal reductions provide us time (T)-, space (S)-, and space-time (ST)-reversal symmetric 


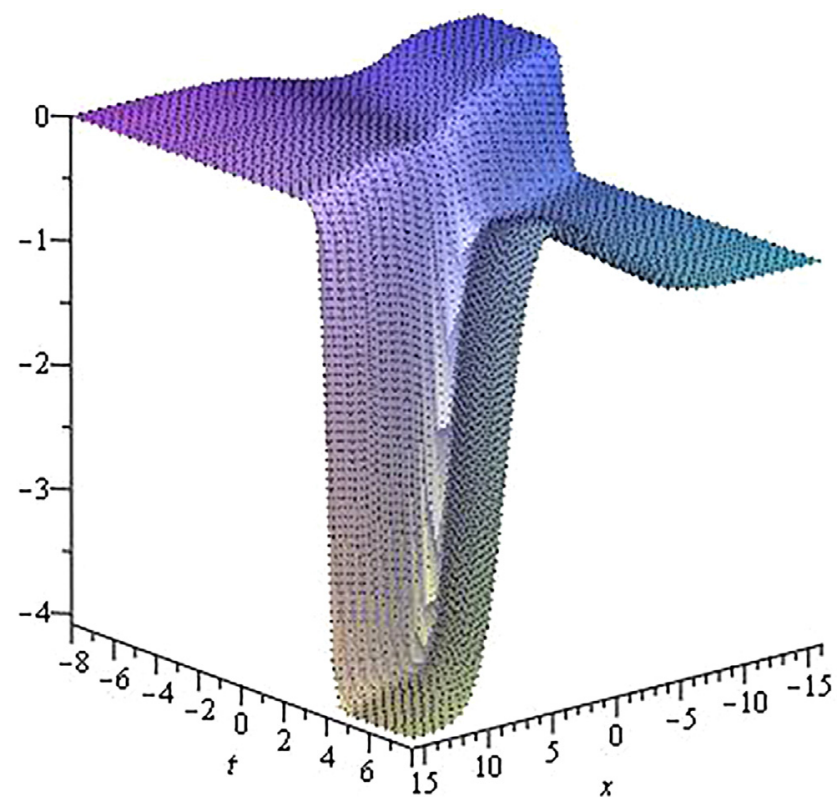

Fig. 12. Soliton and kink-type wave for (120) with the parameters $k_{1}=1, k_{2}=0, \ell_{1}=2, \ell_{2}=\frac{1}{2}, k=-1, a=\frac{1}{4}, \tau_{j}=\rho_{j}=-1, j=1,2$.

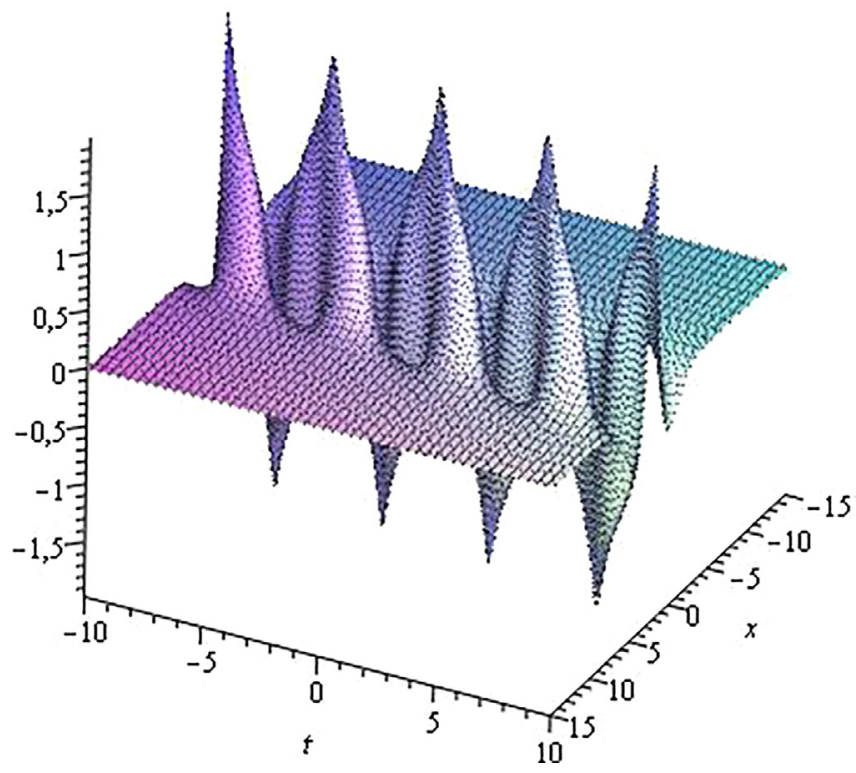

Fig. 13. A breather-type wave plotted for (120) with the parameters $k_{1}=\ell_{1}=1+\frac{i}{2}, k_{2}=\ell_{2}=-1+\frac{i}{2}, k=-1, a=\frac{1}{4}, \tau_{j}=\rho_{j}=1, j=1,2$.

nonlocal cmKdV equations and space-time (ST)-reversal symmetric nonlocal mKdV equation. Each reduced equations are integrable by construction. It means that they have Lax pairs and recursion operators. Starting from any one of the nonlocal cmKdV equations we can generate infinitely many other nonlocal higher order cmKdV equations. Here in this work we mainly focus on the soliton solutions of the nonlocal cmKdV and nonlocal mKdV equations. When we use the Hirota method to obtain soliton solutions of these equations we observed that there are two different types. We present one-soliton solutions of the nonlocal equations of all types but we present only one type of two- and three- soliton solutions. We also plot the graphs of these solutions for particular values of the parameters of the solutions.

From the study of NLS and mKdV systems we observed that they both have local and nonlocal reductions. Moreover in both of these systems there corresponds at least one nonlocal reduction to a local reduction. Both systems have $r(t, x)=k \bar{q}(t, x)$ as local reduction and the corresponding nonlocal reductions are $r(t, x)=k \bar{q}\left(\varepsilon_{1} t\right.$, $\left.\varepsilon_{2} x\right)$ where $k$ is real constant and $\left(\varepsilon_{1}, \varepsilon_{2}\right)=\{(1,-1),(-1,1),(-1,-1)\}$. From these reductions we obtain local and nonlocal NLS equations and local and nonlocal complex mKdV equations. The $\mathrm{mKdV}$ system has additional local and nonlocal reductions. Local reduction 
$r(t, x)=k q(t, x), k$ is real constant, and its corresponding nonlocal reduction $r(t, x)=k q(-t,-x)$ give the nonlocal mKdV equation. From all these experiences we conclude with a conjecture: If a system of equations admits a local reduction then there exists at least one corresponding nonlocal reduction of the same system.

\section{Acknowledgment}

This work is partially supported by the Scientific and Technological Research Council of Turkey (TÜBITAK).

\section{References}

[1] Ablowitz MJ, Musslimani ZH. Integrable nonlocal nonlinear Schrödinger equation. Phys Rev Lett 2013;110:064105.

[2] Iwao M, Hirota R. Soliton solutions of a coupled modified KdV equations. J Phys Soc Jpn 1997;66(3):577.

[3] Tsuchida T, Wadati M. The coupled modified Korteweg-de Vries equations. J Phys Soc Jpn 1998;67(4):1175.

[4] Qi-Lao Z, Zhi-Bin L. Darboux transformation and multi-solitons for complex mKdV equation. Chin Phys Lett 2008;25(1):8.

[5] Hirota R. Exact solution of the modified Korteweg-de Vries equation for multiple collisions of solitons. J Phys Soc Jpn 1972;33:1456.

[6] Ablowitz MJ, Kaup DJ, Newell AC, Segur H. The inverse scattering transform-fourier analysis for nonlinear problems. Stud Appl Math 1974;53(4):249.

[7] Ablowitz MJ, Musslimani ZH. Inverse scattering transform for the integrable nonlocal nonlinear Schrödinger equation. Nonlinearity 2016;29:915.

[8] Ablowitz MJ, Musslimani ZH. Integrable nonlocal nonlinear equations. Stud Appl Math 2016;139(1):7.

[9] Fokas AS. Integrable multidimensional versions of the nonlocal Schrödinger equation. Nonlinearity 2016;29:319.

[10] Sakkaravarthi K, Kanna T. Bright solitons in coherently coupled nonlinear Schrödinger equations with alternate signs of nonlinearities. J Math Phys 2013;54:013701.

[11] Gerdjikov VS, Saxena A. Complete integrability of nonlocal nonlinear Schrödinger equation. J Math Phys 2017;58(1):013502.

[12] Sinha D, Ghosh P. Integrable nonlocal vector nonlinear Schrödinger equation with self-induced parity-time symmetric potential. Phys Lett A 2017;381:124.

[13] Gerdjikov VS, Grahovski DG, Ivanov RI. On integrable wave interactions and lax pairs on symmetric spaces. Wave Motion 2017;71:53.

[14] Gerdjikov VS, Grahovski DG, Ivanov RI. On the n-wave equations with PT symmetry. Theor and Math Phys 2016;188(3):1305.

[15] Gerdjikov VS. On nonlocal models of Kulish-Sklyanin type and generalized fourier transforms. Stud Comp Int 2017;681:37.

[16] Khare A, Saxena A. Periodic and hyperbolic soliton solutions of a number of nonlocal nonlinear equations. J Math Phys 2015;56:032104.

[17] Li M, Xu T. Dark and antidark soliton interactions in the nonlocal nonlinear Schrödinger equation with the self-induced parity-time-symmetric potential. Phys Rev E 2015;91:033202.

[18] Huang X, King L. Soliton solutions for the nonlocal nonlinear Schrödinger equation. Eur Phys J Plus 2016;131:148.

[19] Wen XY, Yan Z, Yang Y. Dynamics of higher-order rational solitons for the nonlocal nonlinear Schrödinger equation with the self-induced paritytime-symmetric potential. Chaos 2015;26:063123.

[20] Gürses M. Nonlocal Fordy-Kulish equations on symmetric spaces. Phys Lett A 2017;381:1791.

[21] Gürses M. Nonlocal super integrable equations. ArXiv:1704.01273 [nlin.SI] [Preprint]. 2017. cited[2017 Apr 5]: [8p.]. Available from: https://arxiv.org/ abs/1704.01273.

[22] Caudrelier V. Interplay between the inverse scattering method and Fokas's unified transform with an application. Stud Appl Math 2017;140:3.

[23] Yang J. General n-solitons and their dynamics in several nonlocal nonlinear Schrödinger equations. ArXiv:1712.01181v1 [nlin.SI] [Preprint]. 2017 cited[2017 Dec 4]: [11p.]. Available from: https://arxiv.org/abs/1712.01181.

[24] Feng BF, Luo XD, Ablowitz MJ, Musslimani ZH. General soliton solution to a nonlocal nonlinear Schrödinger equation with zero and nonzero boundary conditions. ArXiv:1712.09172 [nlin.SI] [Preprint]. 2017 cited[2017 Dec 26]: [26p.]. Available from: https://arxiv.org/abs/1712.09172.

[25] Ma LY, Shen SF, Zhu ZN. Soliton solution and gauge equivalence for an integrable nonlocal complex modified Korteweg-de Vries equation. J Math Phys 2017:58:103501.

[26] Ji JL, Zhu ZN. On a nonlocal modified Korteweg-de Vries equation: Integrability, darboux transformation and soliton solutions. Commun Non Sci Numer Simul 2017;42:699.

[27] Ji JL, Zhu ZN. Soliton solutions of an integrable nonlocal modified Korteweg-de Vries equation through inverse scattering transform. J Math An and Appl 2017;453:973.

[28] Yang B, Yang J. Transformations between nonlocal and local integrable equations. Stud Appl Math 2017;140:178.

[29] Lou SY. Alice-Bob systems, $P_{s}-T_{d}-C$ principles and multi-soliton solutions. ArXiv:1603.03975v2 [nlin.SI] [Preprint]. 2016 [cited 2016 Apr 10]: [22p.]. Available from: https://arxiv.org/abs/1603.03975

[30] Lou SY, Huang F. Alice-Bob physics: coherent solutions of nonlocal KdV systems. Sci Rep 2017:7:869.

[31] Li C, Lou SY, Jia M. Coherent structure of Alice-Bob modified Korteweg de-Vries equations. ArXiv:1706.08178v1 [nlin.SI] [Preprint]. 2017 cited [2017 Jun 25]:[19p.]. Available from: https://arxiv.org/abs/1706.08178.

[32] Gürses M, Pekcan A. Nonlocal nonlinear Schrödinger equations and their soliton solutions. J Math Phys 2018;59. 051501, arXiv: 1707.07610v1[nlin.SI] [Preprint]. 2017 [cited 2017 Jul 24]: [30p.]. Available from: https://arxiv.org/abs/1707.07610.

[33] Gürses M, Pekcan A. Nonlocal nonlinear modified KdV equations and their soliton solutions. ArXiv:1711.01588v2 [nlin.SI] [Preprint]. 2017 [cited 2017 Nov 25]: [52p.]. Available from: https://arxiv.org/abs/1711.01588. 